\title{
Anticancer Drugs in Liposomal Nanodevices: A Target Delivery for a Targeted Therapy
}

\author{
Giorgia Urbinati $^{1,2, *}$, Véronique Marsaud ${ }^{1,2}$ and Jack-Michel Renoir ${ }^{1,2}$ \\ ${ }^{1}$ UMR CNRS 8203 Vectorology and Anti-Cancer Therapeutics, Institut Gustave Roussy, 114, Rue E. Vaillant, 94805 \\ VILLEJUIF CEDEX, France; ${ }^{2}$ Inserm U1021, CNRS UMR 3347, Institut Curie, Orsay, France; ${ }^{3}$ Inserm U749, Institut \\ Gustave Roussy, 114, Rue E. Vaillant, 94805 VILLEJUIF CEDEX, France
}

\begin{abstract}
For many years, nanocarriers have been investigated to modify pharmacokinetics and biodistribution of various active molecules. In the cancer domain, one of the biggest challenges still remains the improvement of the therapeutic index, often too low, for the majority of antitumor drugs. The application of nanotechnologies for the treatment and the diagnosis of cancers are nowadays currently developed, or under development, and liposomes play an important role in the history of nanodevices. Because of their high degree of biocompatibility, lipid nanosystems have been used to improve pharmacological profiles of various anticancer drugs otherwise discarded because of their low water solubility, poor bioavailability or either fragile and subjected to rapid biotransformations. This review aims at introducing an overview of the last 40 years of liposome researches until the last liposomal formulations commercially available or undergoing clinical trials. Liposome properties will be described, with a particular emphasis over the last generation of carriers appreciated for their active targeting characteristics. Researchers foresee a remarkable impact of nanotechnologies in the field of medicine; this review will try to summarize the main concepts over liposome domain, which can count on encouraging results as target therapy associated with targeted delivery.
\end{abstract}

Keywords: Cancer, EPR effect, immunoliposomes, lipid nanocarriers, pharmacokinetics, targeted delivery.

\section{INTRODUCTION}

The aim of this review is to introduce one of the oldest targeted therapy, taking advantage of nanotechnology which first appeared in the early 1970's and which is nowadays commonly used in clinical therapy as well as still under investigation.

A relative simple concept of packing an active molecule with a lipid component was first described by Johnson in 1969 [1, 2], after Bangham had described the characteristics of lamellae of swollen phospholipids in 1965 [3]. The idea of using liposomes as nanocarriers was then developed and improved by a thousand of laboratories all over the world and applied in varied different fields from cosmetic [4] to cancer treatment, and this review will mainly focus on this latter domain.

Liposomes are defined as vesicles formed by an internal aqueous core well protected from the external environment by one or more lipid bilayers (mostly phospholipids). Once these lipids are in contact with an aqueous media, they spontaneously reorganise themselves forming vesicles able to entrap different molecules in the inner compartment or within the membranes $[5,6]$.

Liposomes membranes are not totally impermeable and the entrapped molecules, depending on their chemical nature, can cross the bilayer more or less rapidly. The choice of the

*Address correspondence to this author at the UMR CNRS 8203 Vectorology and anti-cancer therapeutics, Institut Gustave Roussy, 114, Rue E. Vaillant, 94805 VILLEJUIF CEDEX, France; Tel: +33(0)142113552/5393; E-mail: giorgia.urbinati@igr.fr adequate lipid nanocarrier according to drug properties, in order to modulate the drug release kinetics, will be further discussed in section 2.1.

\subsection{Classification and Most Common Methods for Liposome Preparation}

Lipid vesicles can be divided in different classes depending of their chemical structures or release properties but, the most common classification is based on their size and shape strictly related to their fabrication process.

The size range can vary from $\sim 25 \mathrm{~nm}$ for the smallest nanoparticles up to $1 \mu \mathrm{m}$ for liposomes visible under a light microscope. The structure can include only one single lipid bilayer or multiple concentric membrane stacks separated by a small aqueous volume.

The main categories most commonly used are:

\section{Multilamellar Vesicles (MLV)}

Liposomes are formed by 3 or more concentric lamellae, with a size varying from 100 to $1000 \mathrm{~nm}$. This type of nanocarrier is often chosen to encapsulate lipophilic molecules which are situated within the membrane to prevent as much as possible the loss of materials as a consequence of collision with other membranes or proteins, once in vivo. Their preparation is simple and a scale-up for industrial production is easy to perform. For a laboratory practice, the hand-shaken method is commonly used for this kind of liposomes, and the ultimate size can be adjusted by the extrusion process. They are mechanically stable and can be stored for a long period of time [6]. 


\section{Small Unilamellar Vesicles (SUV)}

They represent the smallest phospholipids vesicles obtainable without loosing the liposomes primary characteristics and maintaining size homogeneity of the population. They are surrounded by only one phospholipidic bilayer and their size is strictly related to the ionic strength of the hydration buffer and the composition of the liposomes. It can vary from $15 \mathrm{~nm}$ for egg lecithin in normal saline solution up to $25 \mathrm{~nm}$ if the dipalmitoylphosphocholine (DPPC) is used as the main phospholipid. Their drug loading efficiency is quite low and the retention properties are spare. Nevertheless, this formulation can be used for molecules tightly bound to the liposomes membranes or when the retention parameter is not important because a rapid in vivo biodistribution in the desired tissue occurs $[5,6]$.

SUV have been well characterized in the past and because of their homogeneity in size and lamellarity; they have been widely employed [7-9]. Their sterilization can be done by filtration over a $0.22 \mu \mathrm{m}$ filter which renders this formulation rapidly ready for an intravenous administration. The preparation method requires a high energy input to produce such a small nanoparticle and precautions are needed to prevent the risk of lipid oxidation, hydrolysis, aggregation and fusion. Bath sonication does not furnish enough energy and ethanol injection leads to an inadequate lipid dilution. French press and probe sonication remain the two methods recommended for their preparation but a scale up for industrial production is, in this case, difficult to realize $[5,6]$.

\section{Intermediate Size Unilamellar Vesicles and Large Unilamellar Vesicles (IUV; LUV)}

IUV present a single phospholipid bilayer and a size between 100 and $200 \mathrm{~nm}$. LUV can reach $1 \mu \mathrm{m}$ in size and are generally used to encapsulate high molecular weight compounds or proteins. A large aqueous volume is entrapped within the lipid barrier, thus water soluble molecules tend to accumulate in high amounts in the inner core, which rends this type of liposomes the most appropriate to encapsulate hydrophilic drugs. LUV, because of their large single membrane, tend to easily break after collision and a partial loss of content is often observed.

Reverse phase evaporation (REV) or double emulsion methods are the most commonly used techniques to make IUV and LUV and a large scale production is easy to achieve.

From the point of view of mechanical stability and retention properties, they are less competitive than MLV, however the active loading of ionizable molecules is enhanced once IUV are used [5, 6]. Fig. (1) represents the most common methods of liposome preparation used in laboratory.

\section{LIPOSOME MAIN PROPERTIES}

Even after almost 40 years of study, liposomes still remain a nanocarrier of interest. The wonder to continue exploring this field is due to their high degree of biocompatibility, since their main structure is made of natural lipids some already present in the body. Moreover, they improve the therapeutic index of encapsulated drugs [10] which renders the nanodevices extremely interesting for the delivery of highly toxic molecules such as those used in cancer chemotherapy.

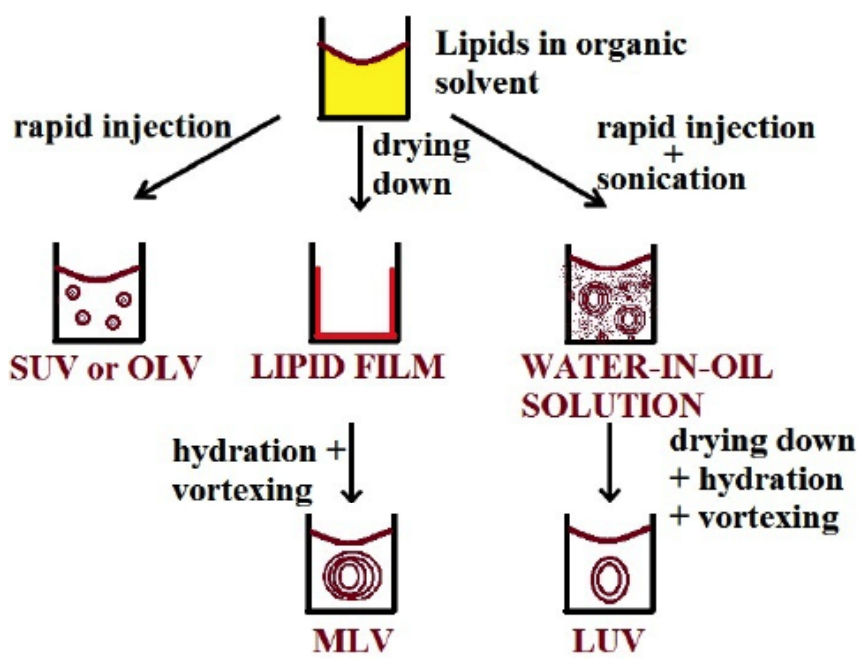

Fig. (1). Most frequently used laboratory preparation methods to obtain lipid nanocarriers with different physicochemical properties.

In this section, we shall discuss the main properties attributable to liposomes and how they can influence the fate of encapsulated drugs.

To obtain a satisfying nanocarrier, it is necessary to take into account the physicochemical properties of the drug chosen to be encapsulated. Based on the active molecule characteristics, the liposome will be conceived with an appropriate lipid composition, size and structure.

\subsection{Drug Criteria Influencing the Choice of Liposomes}

The choice of the right liposomal formulation is extremely important in order to bring beneficial supplies to antitumor drugs. The active molecule needs to be efficiently loaded into liposomes and the nanodevice must keep most of its initial characteristics until it reaches the site of action which means that liposomes must retain their content during the blood circulation and release it only once they accumulate in the target tissue.

Depending on their chemical composition, molecules will tend to distribute differently into liposome compartment and the release behaviour will be strictly related to their distribution into the body [10]. Highly hydrophilic drugs are mostly incorporated in the aqueous core of the nanodevice and the lipid composition of the membrane regulates their retention. In rare cases their maximum loading efficiency can reach $70 \%$ [11], but most of the times it is too low to maintain a drug therapeutic potential once injected in vivo. Moreover, fragile molecules can be subjected to hydrolytic phenomenon once they are stored in the aqueous liposomal compartment, thus a verification of drug stability is recommended before their encapsulation.

Many research groups have proposed to realize some more hydrophobic analogue of water soluble drugs in order to improve their loading into liposomes [10]. Mitomycin C [12], 5-fluorouracil [13], methotrexate [14] have been modified with the addition of lipophilic groups in regions not important for the molecule activity creating then a prodrug 
which was subsequently incorporated into liposomes. After such a modification, the encapsulation yield remarkably raised and the antitumor efficacy increased because a larger quantity of drug was able to be delivered.

The group of Perez-Soler developed a lipophilic cisplatin analogue, the cis-bis-neodecanoato-trans-R,R-1,2diaminocyclohexane platinum (L-NDDP) which was then encapsulated into liposomes reaching a $80 \%$ entrapment efficiency with a subsequent improvement in the cytotoxic activity on cisplatin-sensitive and cisplatin-resistant cell lines [15]. Liposomal L-NDDP is now under phase II clinical trials for the treatment of various types of cancer, such as pancreatic, colorectal and other solid malignancies [16] (see section 4.2.).

Once in presence of highly hydrophobic drugs, the first challenge to think about is how to administrate them since the intravenous route is not allowed and bioavailability is very low. In the past as well as nowadays, pharmaceutical companies invest in chemical research to render insoluble active molecules more hydrophilic in order to facilitate their administration. The use of a liposomal formulation, easily injectable by IV route, can overcome this hinder and let develop some highly active compounds often discarded due to their administration difficulties. Lipophilic drugs tend to interact with the lipid bilayer and there is a relative small drug loss from liposomes during storage [10]. However, since the molecule interacts with the external lipid membrane, a fast redistribution to plasma components can occur once the formulation is IV injected [17]. Multilayers vesicles (MLV) are then the most indicated liposomes to use since the molecules can distribute in different bilayers other than the most external one, being then well protected from the external environment. Lipophilic substances are easily uptaken by vesicles, compared to hydrophilic compounds and the encapsulation yield can reach $100 \%$ which contributes to reduce production cost because no drug loss occurs during the fabrication process. Another important aspect from the commercial point of view is the liposome formulation shelf life. The possibility to freeze-dry the product overcomes most of the stability problems, including aggregation and leaching but, when the powder is re-hydrated, liposomes must reconstitute an undamaged bilayer to prevent drug leakage. As the association between lipids and hydrophobic molecules is stronger compared to hydrophilic drugs, the reconstitution of dried liposomes containing lipophilic drugs leads to appreciable encapsulation efficiency even 24 hours after hydration which means that the drug is still associated with liposomes [18].

One more advantage carried out from liposomes encapsulating lipophilic molecules is their stability over sterilization processes. Parental administrations have to be sterile, thus a sterilization step needs to be foreseen paying attention on preserving liposome structure. Garelli et al. observed complete retention of lipophilic drugs encapsulated into liposomes after autoclaving procedures $[19,20]$ which was not the case of hydrophilic and amphipathic drugs whose leakage was complete (i.e. doxorubicin) [21]. From the point of view of stability, utility and cost, lipophilic drugs have been considered the best candidates for a liposomal formulation [10].
For amphiphilic molecules which tend to easily cross membranes, lipid barrier composition and liposome preparation method play an important role on the stability of formulations [17]. The use of saturated phospholipids such as distearoylphosphocholine (DSPC) or hydrogenated soybean phosphatidylcholine (HSPC) lead to obtain a more rigid bilayer with a consequent better drug retain, essential to maintain a good pharmacokinetics profile after injection in the blood stream.

The studies of Bialer's group give us an example over doxorubicin and its behaviour once encapsulated into lipid bilayer whose composition was mostly based on saturated or poly-unsaturated phospholipids. After IV administration in mice, the egg-phosphatidylcholine (ePC) / cholesterol (Chol) formulation was found to release almost $50 \%$ of the drug within the first hour and $70 \%$ by 4 hours (doxorubicin plasma levels analysis), otherwise when the DSPC replaced the $\mathrm{ePC}$, less than $10 \%$ of the molecule leakage was observed over 24 hours [22]. Moreover, the presence of cholesterol is especially recommended once we deal with this kind of molecules because it helps to reduce their membrane permeability $[23,24]$. In addition, cholesterol seems to stabilize the other lipids present in the bilayer such as phosphatidylcholines (PC) or phosphatidylglycerol (PG) preventing their transfer to plasma lipoproteins (i.e. high density lipoproteins (HDL)) [25]. Introducing a percentage of cholesterol in the lipid composition to prevent drug leakage, is a concept already exploited in the late 1970's when the group of Papahadjopoulos showed that a reduction of cytarabine (Ara C) release was possible once cholesterol was added into the lipid bilayer at $1: 1$ molar ratio [26].

If the drug has an ionizable group, the ammonium sulfate or the $\mathrm{pH}$-gradient loading are the most frequently used liposome preparation methods [27-30]. They take advantage from changing the $\mathrm{pH}$ between the internal core of liposomes and the external environment to favour the molecule crossing within the membrane and block its leak once encapsulated.

The idea is based on the different characteristics that a molecule can assume once it is charged or neutral. For weak bases, an acidic internal $\mathrm{pH}$ is provided while the external $\mathrm{pH}$ is maintained basic. Once the molecule is added, it will be in its uncharged state, then able to cross the lipid bilayer. Once in the internal core, it gets protonated, eventually forming an insoluble salt with the entrapped sulfate ions when the ammonium sulfate is used. The preparation method just mentioned can rise the encapsulation yield of amphiphatic amines up to $100 \%$ [31] and the resulting drug-sulfate gel can increase the liposome stability during storage and after IV injection [32]. The $\mathrm{pH}$ gradient loading method was improved using ionophores like A23187 to prevent the gradient dissipation in order to obtain even more stable formulations [33]. Weak acids can be loaded as well using reverse $\mathrm{pH}$ gradient or calcium acetate instead of ammonium sulfate [34].

The chemical stability of drugs is one of the most important factors to take into account when expecting to use liposomes for a target delivery. If the nanodevice is uptaken by cells through an endocytosis process, the drug will be subjected to low $\mathrm{pH}$ environments such as those present in endosomes or lysosomes and it must be not inactivated by 
these conditions. Houang and co-workers founded a complete inactivation of cytosine arabinoside encapsulated into liposomes after cell uptake [17]. Another example is made by topotecan whose lactone ring is easily hydrolyzed by neutral or basic pHs [35] but, an acidic liposome interior can help stabilize the molecule [36].

Finally, two more important concerns to think about are the drug / lipid ratios that can render the formulation less stable if the ratio results too high [37] and the lipid composition which can lead to lipid peroxidation if the unsaturated lipid percentage is too elevated with a consequent destabilization of liposome membranes [17]. EggPC, for example, contains a high proportion of unsaturated fatty acid chains making it particularly susceptible to oxidation, therefore storage under nitrogen and at low temperatures $\left(4^{\circ} \mathrm{C}\right)$ is recommended.

\subsection{Pharmacokinetics}

The improved pharmacokinetic profiles that liposomes can give to encapsulated molecules after their IV injection are one of the reasons that made these nanocarriers such an interesting formulation for clinical applications. Sometimes, molecules with a therapeutic potential are discarded from further pharmaceutical developments because of their rapid clearance in blood and the consequent need to administrate high doses which can result in toxic side effects. Liposomes can support an increased drug half-life $\left(\mathrm{t}^{1} / 2\right)$ such as in the case of doxorubicin incorporated in ePC / Chol formulation. Doxorubicin's clearance was improved from minutes up to several hours once it was administrated as liposomal formulation compared to the free drug. Even doses of free doxorubicin 3 times higher than those used for respective liposomal formulation could not reach comparable plasma levels [38].

Various are the parameters influencing pharmacokinetics: size, membrane composition, charge, and the way of administration [5]. This section will describe the general factors controlling drug release, and further ahead the specific pharmacokinetics associated with each liposome type will be discussed.

Seniors and co-workers, already in 1985, described the influence of size for the uptake of nanodevices by the reticuloendothelial system (RES). The diminution from $400 \mathrm{~nm}$ to $200 \mathrm{~nm}$ in diameter can reduce the clearance by 7.5 times and SUV particles, with a $25 \mathrm{~nm}$ size, are even 5 times more stable in blood circulation [39].

The charge of lipids can influence as well the clearance by a size-mediated mechanism; for neutral lipids, a possible liposome aggregation can occur with a consequent size increase. Previous studies suggested that the optimal range to reduce at most the clearance and to maintain an efficient

encapsulation yield is around $100 \mathrm{~nm}$ [17]. Aggregation can be avoided introducing charged lipids, but, the consequent pharmacokinetics profile is strictly related with the single lipid characteristics [40] and the relationships between the molecule half-life and the introduction of a charged lipid is extremely complicated [17]. Thus, an accurate study substituting the lipid and maintaining invariable size, surface charge density and all the other factors influencing liposome properties, need to be conducted before making any comparison. For instance, anionic lipids like phosphatidylserine or phosphatidylglycerol are well known to promote a rapid uptake by RES [41], by contrast, the monosialoganglioside (GM1) and phosphatidylinositol prolong the circulation lifetime of negatively charged liposomes compared to neutral liposomes. However, in the latter case, a sterically stabilization due to GM1 can be the cause of such an increased blood residency, a property that will be better described in the "long-circulating liposomes" section. The mol \% of charged lipid used for the fabrication is one more parameter to take into account. As a matter of fact, when an anionic lipid such as distearoyl-phosphoglycerol (DSPG) is added as the main component of liposomes, the circulation half-life is quite reduced [41], but once it is used only as a small fraction (< $10 \mathrm{~mol} \%$ ), it shows an improvement in the pharmacokinetics profile [42].

Another important aspect influencing nanocarriers $t^{1 / 2}$ is the membrane permeability and packing related with the rigidity properties of lipids used. Bilayer fluidity which is strictly related with the nature of lipids can show a relevant impact on the clearance of liposomes and relative encapsulated drug [17].

When lipids whose transition phase temperature (Tm) is significantly above $37{ }^{\circ} \mathrm{C}$ are used as main components for membrane packing, liposomes show a longer circulation lifetime. This is the case of DSPC and HSPC whose Tm is around $15-17{ }^{\circ} \mathrm{C}$ above body temperature and confers a more rigid structure to the lipid bilayer, improving the liposome stability [41]. EggPC or dipalmitoylphosphocholine (DPPC) give a more flexible framework to nanocarriers which can be rendered less permeable thank to the presence of cholesterol [43]. Moreover, sphingomyelin can further reduce liposome clearance. Indeed, this component can form intermolecular $\mathrm{H}$-bound with molecules of cholesterol present in the bilayer rigidifying the nanodevice structure resulting in a decreased plasma protein adsorption on liposome membranes [44].

All these parameters show how the choice of lipid composition can influence the pharmacokinetics behaviour without in any case neglecting the importance of the drug molecule characteristics such as amphiphatic drugs which need high phase transition phospholipids to prevent their leakage from liposomes.

One of the factors that mostly affect the pharmacokinetics profile is the steric stabilization made by compounds such as polyethylene glycol (PEG) and GM1 which gave rise of a new generation of nanocarriers called "long-circulating liposomes", because of their prolonged circulation lifetimes. This second generation of nanoparticles with the mechanism by which the steric hindrance can influence their stabilization in the circulation will be better described in section 3.2.

\subsection{Biodistribution}

Drug accumulation in the desired environment, avoiding healthy tissues, is a fundamental property always researched for target therapies and delivery.

When a free anticancer drug is injected in the blood stream, it is largely distributed in all tissues and if this behaviour is combined with a rapid clearance, the result is a 
low accumulation in the tumor and a significant toxicity in healthy tissues. However, when active molecules are encapsulated into liposomes, their biodistribution is largely modified and, in many cases, improved to the target tumor tissue. The morphology of healthy vessels contributes to reduce the accumulation of such a nanodevice in healthy organs because their relatively large size ( $25-200 \mathrm{~nm}$ ) do not permit to cross the endothelial barrier whose pores are not larger than $2 \mathrm{~nm}$ or $6 \mathrm{~nm}$ in post capillary venules [45, 46] Fig. (2B). On the contrary, liposomes tend to accumulate where the vessels are discontinuous such as in the case of tumors neoangiogenesis, kidney or in organs containing RES macrophages such as liver and spleen reducing a wide healthy tissue distribution and a consequent wide toxicity. Moreover, kidney glomerulus present junctions of 40-60 nanometers in diameter and most of liposomes have a size much higher to permit their glomerular filtration. Interestingly, liposome accumulation into liver is mostly restricted to Kupffer cells which are responsible of external bodies removal, and a low accumulation into hepatocytes is noticed suggesting a less damage of liver tissue than if liposomes accumulate into all liver cells [47]. Factors influencing particulate retention by the spleen are strictly related to surface charge and surface properties. For instance, large surface area, a rigid membrane and a low negative charge can render liposomes similar to reticulocytes, cells that are preferentially uptaken by the spleen to undergo their final maturation step [48].

By contrast, tumors present a higher vascular permeability which depends on various growth factors such as VEGF which is a vascular permeability factor as well as an angiogenic factor helping to create new blood vessels, fundamental to bring new nutriment substances to tumor tissue. The availability of these factors is not homogeneous in all types of cancer which lead to dissimilar tumor microenvironments with a consequent nanocarrier accumulation which is proper to each disease [17]. Moreover, tumors show compromised lymphatics (neolymphangenesis) in combination with a high interstitial pressure. The presence of an impaired lymphatic system, unable to drain out most of the substances accumulated, helps to retain the nanosystem longer than in other tissues where the lymphatic vessels are fully functional [49, 50].

The combination of tumor leaky vasculatures and not fully functional lymphatic system, leads to a higher liposomal drug accumulation in the desired tissue, a phenomenon usually called enhanced permeability and retention effect (EPR) Fig. (2A). The liposome tumor extravasation phenomenon was demonstrated for the first time by Huang and co-workers using gold-labelled liposomes to target Kaposi's sarcoma [51]. Transcytosis pathway is considered less frequent and the liposome accumulation mostly occurred in the tumor interstitium or in resident tumor macrophages, but not within tumor cells [50]. An improved targeting not only of tumors but more specifically of cancer cells within the tumor will be the matter of the section "ligand-targeted liposomes" where the concept of enhancing the tumor cells uptake by binding a specific molecule on liposome surface will be described.

The extent of tumor drug accumulation is mostly related to tumor type, liposome formulation and administered dose. Large tumors are more difficult to treat because of their higher interstitial pressure which does not allow nanosystem to penetrate deeply into the tumor to reach the necrotic area [52]. Very small tumors $(<2 \mathrm{~mm}$ in diameter) as well do not present the typical essential characteristics to permit liposomes to accumulate and release the encapsulated drug because in most of the cases they are avascular [53]. Already in 1988, Papahadjopoulos' group observed a tight correlation between improved circulation lifetimes and higher liposome tumor biodistribution [54], a concept subsequently delved by
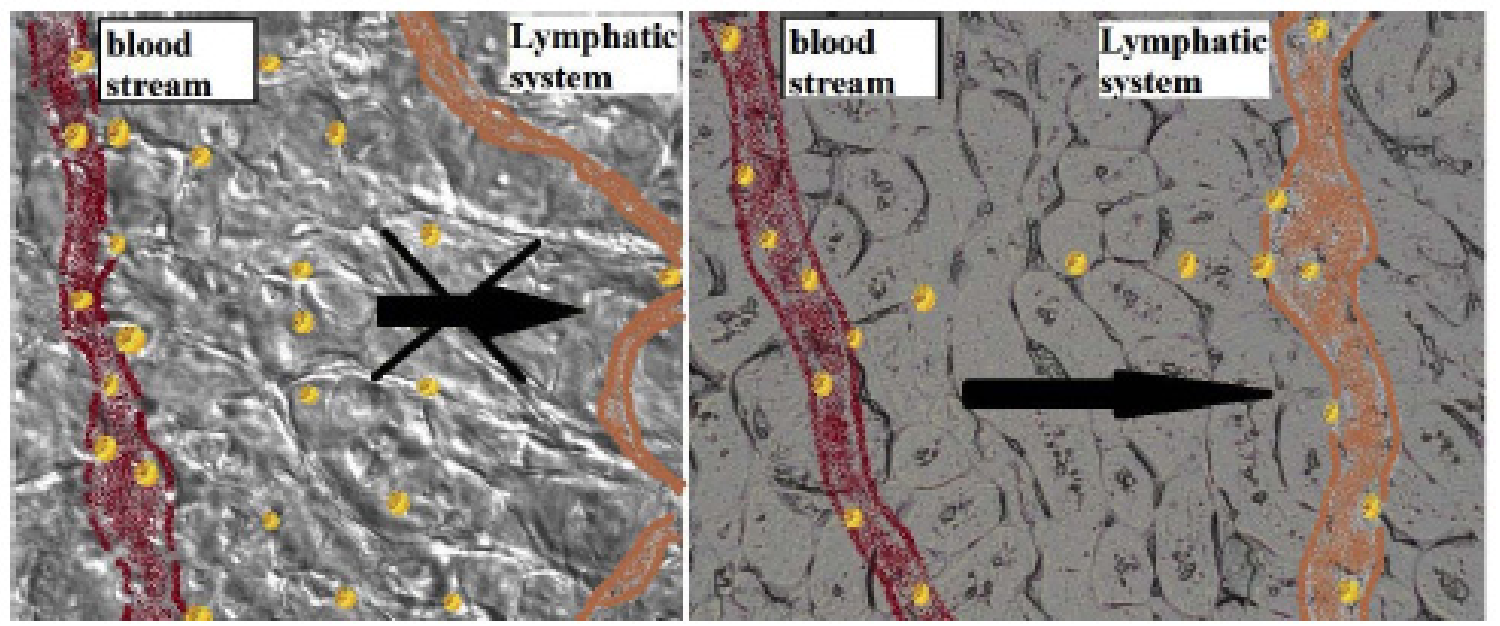

Fig. (2). Enhanced permeability and retention effect (EPR). Panel A represents a microenvironment typical of tumor tissues (MCF-7 breast cancer cells - Renoir's unpublished work) with leaky blood vessels and an impaired lymphatic system unable to drain out of the tissue undesired compounds. Panel B shows a healthy tissue presenting continuous blood vasculatures and preserved functionally active lymphatic vessels. After IV injection, once liposomes (yellow vesicles) reach tumor tissue they are able to extravasate because of the discontinuous blood system and accumulate because the lymphatic circulation is irregular thus unable to remove them. On the contrary, in healthy tissues extravasation of nanocarries is less frequent. Tight junctions present on the endothelium vessel do not allow any permeation and the few nanoparticles able to leave the blood stream are rapidly captured by the fully functional lymphatic system and drained away. 
Gabizon and co-workers analyzing the different behaviour between classical liposomes and long-circulating nanocarriers. If liposomes are able to retain the encapsulated drug for a longer period, they will have the time to accumulate in tumor tissue and release the drug at a higher rate. Mayer's group showed that the different biodistribution between classical liposomes and long-circulating liposomes is not related to the rate but to the time of accumulation which means that conventional liposomes can accumulate into desired tissue, but they are retained for a shorter time, otherwise, longcirculating liposomes show a prolonged tumor distribution which lead to a prolonged drug efficacy over time [55].

Liposome size is one more parameter considered important to improve liposome accumulation in target tissues and in particular to reduce spleen uptake. It is well known that for equivalent formulations, increasing particle size enhance splenic uptake and for size comparable liposomes $(200 \mathrm{~nm})$, a high percentage of cholesterol $(\sim 50 \% \mathrm{~mol})$ can show a stronger affinity for this organ [48].

An increased tumor drug accumulation can be reached associating local hyperthermia to liposome administration [56]. Microvasculature permeability seems to be enhanced when hyperthermia is applied and tumor distribution of liposomes results to be more uniform with an increased bioavailability of the encapsulated drug within tumor cells [17]. Moreover, thermosensitive liposomes can be formulated using lipids such as DPPC whose transition temperature $(\mathrm{Tm})$ is $38{ }^{\circ} \mathrm{C}$. The resulting liposomes are stable at body temperature, and do not release the encapsulated drug. However, when the target tissue is heated at $42{ }^{\circ} \mathrm{C}$, the nanocarrier release the associated drug with a consequent improved drug accumulation where the heat is applied [57].

\subsection{Circumventing Multidrug Resistance (MDR)}

In oncology, one of the biggest challenges to deal with still remains prevention and treatment of tumors insensitive to most commonly used anticancer drugs or the development of drug resistance during chemotherapy. To understand how liposomes can possibly circumvent MDR, its mechanism should be briefly described.

Multidrug resistance is a complex process conferring to cancers a state of resilience to the treatment which can be intrinsic or acquired after antitumor drugs exposure [58]. More than 13 ATP-binding cassette (ABC) transporters were found to participate to MDR phenomenon [59] and Pglycoprotein (P-gp), one of the primary identified drug efflux pumps, is the best characterized protein of the different $\mathrm{ABC}$ transporter [60]. Many other $\mathrm{ABC}$ transporters are involved in MDR process such as multidug resistance protein1 (MRP-1, ABCC1), breast cancer resistance protein (BCRP, ABCG2) and ABCB6 [61, 62]. BCRP has been found responsible of cell resistance to classic antitumor drugs like mitoxantrone, and $\mathrm{ABCB} 6$ associated with the cisplatin and paclitaxel efflux from chemotherapy insensitive cancer cells [63].

Various are the mechanisms involved in the MDR process including pump drug efflux, particularly associated to hypoxia tumor conditions, but also decreased drug influx and enhanced drug metabolism of internalized drugs. Enhancement of P-gp protein expression was observed after hypoxic cell exposure in a time-dependent manner and an upregulation of enzymes such as cytochrome P450, responsible for the metabolism and inactivation of internalized drug as well as activation of glutathione-mediated reduction pathway was noticed $[60,64]$. Moreover inhibition of tumor suppressor $\mathrm{p} 53$, inactivation of pro-apoptotic BAX and BAK factors, activation of anti-apoptotic Bcl-2 proteins and over expression of growth factors (HER-2, EGFR1) were found to be strictly correlated to MDR onset $[65,66]$.

Many authors have suggested that liposomes, and nanocarriers in general, can partially overcome drug resistance but the mechanism responsible of this phenomenon has not been completely elucidated $[64,67,68]$. The use of negative charged phospholipids may regulate the P-gp protein transporter as described by Oudard already in 1991 [69] or endocytosis, a classical process for liposome cell uptake, may deliver antitumor drug bypassing P-gp transporters whose location is in plasma membrane $[68,70]$. Moreover, the high level of drug delivered by liposomes for longer periods compared to free drugs could be the cause of an improved sensitivity of resistant cells to classical non-responding chemotherapy [71].

Interestingly, MDR cells often present a decreased internal $\mathrm{pH}$, a property that can be exploited using $\mathrm{pH}$-sensitive liposomes for an efficient delivery of anticancer drugs. This concept has been already used by Lee and co-workers to deliver doxorubicin-loaded micelles to resistant MCF-7 tumors proving a dramatic cytotoxicity increase in MDR breast cancer cells (pH 6.8) as compared to classic MCF-7 (pH 7.4) [72]. A similar behaviour should be expected from $\mathrm{pH}-$ sensitive liposomes.

Finally, a strategy often used to overcome drug resistance is the combination of an anticancer drug with a MDR modulator. Liposomes combining both molecules in the same formulation was the matter of Wu's work where doxorubicin and, the calcium-channel antagonist, verapamil were encapsulated into trasferrin-active targeting liposomes. In MDR leukaemia cell line, $\mathrm{IC}_{50}$ drastically decreased when targeted liposomes where administered compared to free doxorubicin [73].

Many studies proved the increased efficacy of liposomal anticancer drugs over MDR cells compared to free drug [7476], and various theories have been developed to explain the mechanism by which liposomes are able to overcome MDR (Table 1), nevertheless further investigations are needed to deeply understand this phenomenon.

\section{LIPOSOME DEVELOPMENT OVER THE LAST 40 YEARS}

Since their first appearance in the early 1970 's, the research and development of liposomes never stopped. Their interesting properties of biodegradability, ease of preparation and their potential as carriers for lipophilic molecules, made researchers continue to improve this simple formulation to obtain nanodevices even more efficient, stable, and highly specific for target tissues.

In this section it will be discussed the evolution of these colloidal systems including their pros and cons for each generation. 
Table 1. Use of Liposomal Drug Encapsulation to Limit and Circumvent Multidrug Resistance

\begin{tabular}{|l|l|l|}
\hline - $\quad$ Liposomal anticancer drugs in combination with Pgp inhibitors & MDR inhibitors loaded liposomes can improve anticancer drugs efficacy \\
\hline - $\quad$ Delivery of hydrophobic molecules by liposomes & Substrates for Pgp proteins are mostly hydrophilic \\
\hline - $\quad$ Gene delivery by liposomes & Anionic lipids have shown inhibitory properties against Pgp \\
\hline - Use of pH sensitive liposomes & $\begin{array}{l}\text { Lipoplexes containing antisense oligonucleotides against MDR1 gene (Ala- } \\
\text { hari } \text { et al. 1996) }\end{array}$ \\
\hline - Highly charged liposomes & MDR cells present a lower pH compared to healthy cells \\
\hline - Receptor-mediated uptake of targeted liposomes & $\begin{array}{l}\text { High levels of drug delivered by liposomes for longer periods compared to } \\
\text { free drugs, could be the cause of an improved sensitivity }\end{array}$ \\
\hline
\end{tabular}

\subsection{Conventional Liposomes}

Conventional liposomes, also known as the first generation of liposomes, are typically composed of a high variety of lipids Fig. (3), mainly containing phosphatidylcholine and a percentage of cholesterol to stabilize the lipid membrane. Optimized systems generally contain DSPC and Chol in $55: 45$ or $66: 33$ molar ratio or egg yolk (ePC/Chol) in a 3:2 ratio [17]. Their pharmacokinetics and biodistribution are strictly related to their size, membrane composition and surface charges as previously described in section 2 .

The major limitation for this formulation, as for all pharmaceutical nanocarriers, is their recognition as foreign particles by the defence organs (RES) in the body, conducting to macrophage activation and their fast elimination before completion of any function [77]. Indeed, conventional liposomes are cleared from the blood stream by macrophages mainly present in the spleen and liver. A phenomenon of opsonization, consisting in adsorption of serum proteins such as $\beta 2$-glycoprotein $\mathrm{I}$, complement $\mathrm{C} 3 \mathrm{~b}$ fragment, and other $\mathrm{Fc}$ fragments of $\mathrm{IgG}$ on the lipid membrane surface, is thought to be responsible of recognition by RES macrophages [78]. Their relative rapid clearance is known to be dose-dependent suggesting a saturation of the uptake mechanisms. Likely, macrophages decrease their phagocytic capacity after ingestion of high quantities of lipids, moreover plasma opsonins become saturated thus unable to bind larger amounts of liposomes [17]. Taken together these rudiments outline how important is the administered dose in the lifetime of the nanocarrier but the saturable non-linear kinetics of conventional liposomes complicates the calculation for clinical dosages [79].

Even if classical liposomes are subjected to rapid clearance, and their release kinetics are faster compared to the $2^{\text {nd }}$ and $3^{\text {rd }}$ generation of liposomes they still maintain their property as controlled-release systems which can improve drug pharmacokinetics and biodistribution after IV injection if we compare them to free anticancer drugs [77, 80, 81].

One more issue for this first generation of liposomes is their tendency to aggregate and flocculate over time, then unless they undergo freeze-dry processes, they tend to have a short shelf-life. Small, neutral conventional liposomes could appear modestly efficient as drug delivery vehicles if we take a look over the next generations, anyway they still keep a relevant clinical potential for the treatment of liver and spleen cancers. As they preferentially accumulate in these organs in a rapid and specific manner, they would be the formulation of choice over other more complex liposomes.

Indeed, various conventional liposomes were approved by the FDA and are commercially available for the treatment of different types of cancer such as DaunoXome ${ }^{\circledR}$ for Kaposi's sarcoma, Myocet ${ }^{\mathbb{B}}$ as combination therapy for recurrent breast cancers as well as for the treatment of other pathologies like Ambisome ${ }^{\circledR}$ for fungal infections and visceral leishmaniasis [77].

\subsection{Long-Circulating Liposomes}

One of the reasons that made liposomes still nowadays the matter of investigation for delivering new molecules and new targeted therapies was the improvement of their blood stream residency thank to surface modifications first introduced in the early 1990's [82, 83]. Hydrophilic polymers showed the ability to protect colloidal systems from interaction with different solutes and this protection phenomenon has been called "steric stabilization" [84]. The two molecules with major success were the monosialoganglioside GM1 and the polyetyleneglycol polymer (PEG) $[85,86]$. In the latter case PEG was chemically attached to phospholipids through a laboratory synthesis [87]; later PEG-phospholipids were commercially available favouring the use of this polymer over GM1. The typical composition of sterically stabilized liposomes is made of 4-6 \% of DSPE-PEG $2000,30 \%$ of cholesterol and the remainder of saturated or unsaturated phospholipids [17]. As previously mentioned, PEGylated liposomes (also known as Stealth ${ }^{\circledR}$ liposomes) showed an enhanced circulation half-life which was demonstrated by a wide number of different laboratories on various type of animals $[54,87,88]$. Initially, a theory over a decreased opsonization process has been found to explain the longer circulation lifetime of this second generation of liposomes. After a large number of studies over molecular weight of PEG chains and PEG mol percentage to use for an optimized formulation, the approved composition was found to be made of $5-10 \mathrm{~mol} \%$ of PEG and with PEG length varying from 2.000 to 5.000 Dalton.

The concept is based on the flexibility properties of PEG chains or GM1 which occupy the space immediately adja- 
cent to liposome surface and the possibility to create a hydration shell around the particles hindering interactions with other components [89] Fig. (3). A consequent decrease in surface adsorption of plasma proteins, opsonins and other blood components should occur, thereby interactions with RES macrophages are inhibited [90-92]. A common misconception is that PEG introduction in liposome composition totally avoid RES uptake whereas the macrophage interaction is simply reduced and the accumulation in these tissues is slower compared to classical liposomes [93].

However, further studies highlighted that prolonged lifetime of sterically protected liposomes in the blood stream may not be only the consequence of a reduced protein adsorption; on the contrary opsonization of stealth liposomes frequently occurs [94-97]. For instance, Szebeni and coworkers showed a significant complement activation in human serum when liposomes presenting 5 mol \% of PEG-PE were introduced [98]. Various theories have been developed taking profit of these new breakthroughs: firstly, even if the complement fixation on PEG chains occurs, its location could be inaccessible for complement-receptors recognition. Secondly, the cleaved complement fragment (C3bn) surface bound may interact with the complement receptor 1 (CR1) present on erythrocytes explaining the prolonged blood residency [99]. Furthermore, the physiological state of macrophages seems to play a significant role in nanoparticles phagocytosis. Under normal physiological conditions, the macrophages receptors, responsible of nanocarriers recognition, are generally blocked by dysopsonins, whereas in pathological environments, such as in tumors, macrophages are in their activated state and then able to phagocyte stealth liposomes [100].

An understanding of the mechanisms responsible for maintaining high blood levels of long-circulating nanoparticles is essential to optimize the circulation profiles of these nanosystems.

Sterically stabilized liposomes present nonsaturable, loglinear kinetics [101] which offers the unique advantage to be dose-independent [79] and relatively insensitive to nanovectors size, charge and lipid composition [102].

The major improvement for this new generation of liposomes, beside their long circulation lifetime, is then the acquired relative independency of formulation from most of the physicochemical characteristics compared to classical liposomes. Thus, since size, phospholipids compositions and surface charges play a less important role in pharmacokinet-

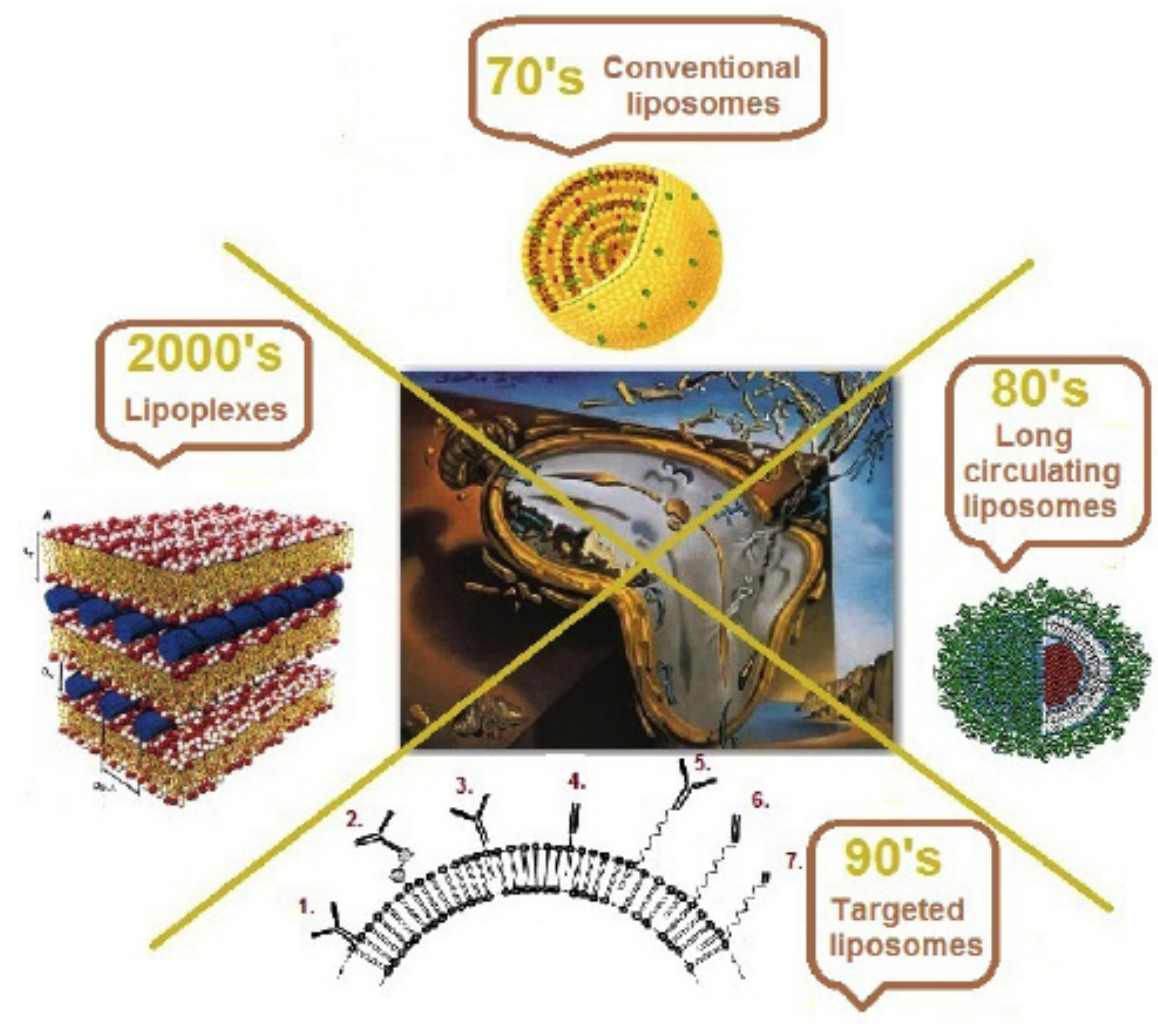

Fig. (3). Overview of the last 40 years in liposome evolution. During the 1970's a simple lipid shell containing an active molecule was usually performed. In the ' $80 \mathrm{~s}$, liposome pharmacokinetics have been improved thank to hydrophilic polymers grafted on the surface of liposomes. This improvement made them called long-circulating or sterically stabilized liposomes. The last decade is known as the decade of the targeted therapy, active targeted liposomes as well as immunoliposomes have been developed for a wide range of cancer applications. 1 . represents whole antibody simply adsorbed on liposome surface; 2. represents whole antibody-hapten binding technique; 3 . represents a covalent binding between whole antibody and liposome surface; 4. represents Fab' antibody fragment covalently attached on the surface of liposome; 5. represents a covalent binding of whole antibody on the extremity of PEG chains; 6. represents a covalent binding of Fab' antibody fragment on the extremity of PEG chains; 7. represents the last generation of immunoliposomes containing a scFv fragment covalently linked to liposomes PEG chains. The new millennium has seen the birth of the last generation of liposomes containing siRNA, oligopeptides, DNA or plasmids (lipoplexes). 
ics and biodistribution, the studies can be focused on a more open wide range of formulations that can be shaped in a better specific manner according to the chemical characteristic of the selected drug. The rate-limiting step for Stealth ${ }^{\circledR}$ liposomes clearance becomes the drug leakage which can be improved with more flexibility in the choice of liposomes structure.

Accordingly to tumor micro-environment previously described in section 2.3., colloidal systems able to circulate for longer periods in the blood stream should accumulate in the tumor target tissue at higher rates [103, 104]. This was the challenge when the first sterically stabilized liposomes were conceived and several studies have been carried out comparing tumor drug accumulation delivered by classical or Stealth $^{\circledR}$ liposomes $[50,55,105]$. The first observations showed that conventional liposomes may accumulate in cancer tissues at a more rapid rate than long-circulating liposomes, however a lower accumulation was seen for longer extents periods of time ( $4 \mathrm{~h}$ vs. $24 \mathrm{~h}$ ) [55].

An interesting assessment made by Drummond in favour of sterically stabilized liposomes over classical liposomes is the difference in the rapidity of tumor growth between animal models and humans. In the first case tumors have doubling times of days or weeks whereas in humans are mostly in the range of weeks to months. For humans, the rapidity in drug accumulation is then less important, such as in the case of conventional liposomes, rather than the overall drug flux extended over days after administration which is the case of long-circulating liposomes able to accumulate within the tumor for longer periods of time [17]. The enhanced drug therapeutic efficacy of this second generation of liposomes was observed by different laboratory groups [26, 106]. For example, doxorubicin, one of the most studied drugs for liposome formulations, enhanced significantly its therapeutic efficacy once encapsulated into Stealth ${ }^{\circledR}$ liposomes compared to either free and conventional liposomal drug and this result could be observed in many different types of cancer [107, 108]. One more advantage of sterically stabilized liposomes is their drug leaking rate generally inferior compared to classical liposomes and during storage since PEG chains provide a steric barrier that prevents aggregation phenomenon [17, 109, 110].

Thank to their high versatility for the encapsulation of a wide range of antitumor drugs, Stealth ${ }^{\circledR}$ liposomes are nowadays exploited for many different cancer therapies. Few examples of the recent works are: the modified pharmacokinetics and biodistribution of liposomal Oridonin, an anticancer drug presenting cardiac toxicity [111]; the first clinical trail of liposomal CKD602 formulation in patients with refractory solid tumors [112]; new 4-hydroxy-tamoxifen-loaded $\mathrm{pH}$ gradient liposomes as therapeutic potential in a multiple myeloma experimental model [113].

This second generation of liposomes made an overall improvement in liposome field [114], nevertheless the tumor accumulation is made by a passive targeting which lead to a drug release within the stroma and the interstitial space of tumors but not specifically to cancer cells. For small lipophilic molecules, able to diffuse through cell membranes, this process is not an issue, but when aiming at delivering high hydrophilic drugs or high molecular weight compounds, the non specific cell delivery can become a failure.

\subsection{Ligand-Targeted Liposomes}

In parallel with the development of long-circulating liposomes, a new approach has emerged to create nanosystems able to target drugs not only to tumor tissue but more specifically to tumor cells Fig. (3). In the last decades cancer research focused on finding new molecular features specific of tumor cells such as over expression of some receptors or down regulation of gene suppressors. Taking advantage from these last discoveries, researchers in the field of nanomedicine could conceive colloidal systems whose accumulation in the desired tissue was not only due to physical properties, such as the EPR effect (passive targeting), but also more specifically due to interaction with cancer molecular targets (active targeting).

Ligand-directed liposomes provide true intracellular drug delivery thank to an internalization process. Once the drug is released directly into the cytoplasm, it can potentially circumvent membrane-bound efflux mechanisms permitting a higher drug cell accumulation [64]. However, some considerations must to be taken into account when designing such systems: i) the attachment of the ligand must not modify pharmacokinetics and biodistribution of the nanocarrier; ii) ligand-targeted liposomes must avoid any unwanted immune response; iii) the ligand amount coupled on the surface of liposome has to be deeply investigated in order to allow target binding without loosing any long-circulating property [85].

The improvements made over ligand-targeted liposomes during the past decade and the variety of ligands used in the past and nowadays for this third generation of liposomes will be discussed in the following section.

Many different attempts have been made to optimize targeted-liposomes and various researches in this field are still going on. The major developments have been made on the attachment of antibodies on vesicles surface (immunoliposomes), but other molecules as well have been used as target and will be described later on. Weissmann and coworkers were the first investigating on the methods of promoting macromolecules delivery into cells using liposomes [115]. They adsorbed non-specifically antibodies over preformed vesicles by a heating process, and the result was an enhanced phagocytic uptake resulting in improved macromolecule cell internalization [116]. However this approach lacked of precision, the antibody amount, specificity and stability were difficult to control and hydrophobic interactions between immunoglobulin and vesicles were too weak to maintain stability following in vivo administration [116].

Other groups tried to develop more controlled approaches [117] and the outstanding breakthrough was made by Papahadjopoulos' team when he proposed irreversible coupling of immunoglobulin fragments to preformed vesicles [118]. The essential improvements were made by the irreversible bound which was stable in serum compared to previously used disulfide bounds [119] and the coupling of the Fab' fragment instead of the whole antibody. 
Indeed, while developing which was the best method to attach ligands to nanocarriers, another field of investigation was to understand how to decrease immune responses and improve long-circulating properties.

Although immunoliposomes demonstrated an improved in vitro specificity, their in vivo efficacy was highly limited. Investigations outlined an augmented uptake by RES organs with a consequent rapid liposome removal from blood circulation. Two different approaches were then carried out to overcome such obstacle, one consisting in combining PEG and antibodies, and the other developing Fab' and single chain fragments $(\mathrm{scFv})$ issued from immunoglobulins. Maruyama et al. demonstrated an improved circulation half-life when PEG chains were introduced on the surface of liposomes, but PEG shielded liposomal ligands preventing an efficient binding $[88,120]$. Allen and co-workers proposed an option to circumvent this issue attaching the immunoglobulins at the distal end of PEG chains providing them a significant freedom of movement and preserved ability to bind targets $[121,122]$. The two approaches of PEG and Fab or $\mathrm{scFv}$, use were later cleverly combined obtaining so called third generation of liposomes [123, 124].

Since immunoliposomes and ligand-targeted liposomes were optimized in term of circulation lifetimes and lipid/antibody ratio assuring specific targeting [125], the last decade has shown the development of a large variety of different liposomes taking advantage from this new generation. The rest of this section will describe ligands and antibodies mostly used and having a possible future in clinical therapy.

One of the oldest and best studied targeted nanocarrier is undoubtedly folate-modified liposome since folate receptor is well known to be over expressed in many cancer cell lines [126-129]. Even if this approach is still widely exploited [130], folic acid still remains a nutriment compound for tumor cells, thus targeting a system with a molecule promoting cell proliferation is not fully recommended.

Transferrin (Tf) receptor (TfR) is equally highly expressed in tumor tissues, antibodies against the receptor as well as transferrin itself were used as ligands for an active targeting of liposomes [131] and for gene delivery through cationic liposomes [132].

Vasoactive intestinal peptide (VIP)-targeted liposomes encapsulating radionuclides were used for breast cancer imaging and authors suggest a probable enhanced anticancer effect once a antitumor drug is associated [133, 134].

Moreover, arginine-glycine-aspartate peptides (RGD) coupled on the surface of liposomes were used to target integrins present in tumor vasculatures and once the colloidal system was loaded with doxorubicin and combretastatin A-4, it demonstrated an improved efficacy against B16F10 melanoma tumors subcutaneously growing in $\mathrm{C} 57 \mathrm{BL} / 6$ mice [135].

Hyaluronan receptors are as well one of the favourite target for this third generation of liposomes and for nanocarriers in general as well described by Platt and Szocka [136]. Peer and co-workers described the antitumor effects of mytomicin $\mathrm{C}$ loaded liposomes targeted with hyaluronic acid in three different mice tumor models, however, as already mentioned for folic acid, many research articles describe hyaluronic acid as a promoter of cell proliferation $[137,138]$ thus not well indicated for cancer therapy.

The sulfated glycosaminoglycan (GAG), chondroitin, was used as ligand for cisplatin loaded liposomes and showed a successful inhibition of tumor growth and metastases [139]. Trans-activating transcriptional activator protein (TAT) and penetratin have been used to enhance liposome internalization as well [140].

An alternative complementary physical approach consisting in magnetic targeting proved to be highly efficient to guide and accumulate magnetic nanoparticles into selected tissues or cells by applying an external magnetic field gradient [141, 142]. Magnetic-fluid-loaded liposomes (MFL) were elaborated based on 200-nm unilamellar PEGstabilized phospholipids vesicles which encapsulate a physiologically compatible suspension of nanocrystals of maghemite $\left(\gamma-\mathrm{Fe}_{2} \mathrm{O}_{3}\right)$ [143]. They can be magnetically driven by an external magnet towards solid tumors subcutaneously implanted in mice, without no damage to the lipid bilayer and no leakage of the internal aqueous content. Indeed, a magnetic field gradient significantly enhances MFL uptake by human cancer cells $[144,145]$. However, the efficiency of such a magnetic liposome is limited to external solid tumors.

Among antibodies used for active targeting, anti-HER2 and anti-CD 19 are surely the two most exploited immunoglobulins coupled on the surface of liposomes. Two major liposome research laboratories, Papahadjopoulos', now become Park's group, and Allen's group were the pioneers in this field and their works seem to be the most promising for an early future development in clinical trials. Indeed, since many years anti-HER-2, also known as Herceptin ${ }^{\circledR}$ (trastuzumab) is FDA approved and commercially available for the treatment of breast cancers over expressing HER2 receptors. Anti-CD 19 is as well in clinical trials for the treatment of leukaemia and lymphoma. Hence, since several works proved the real antitumor improvement of $\mathrm{scFv}$ against $\mathrm{CD}$ 19 or ErbB2 bounded on the surface of liposomes [125, 146148], and these antibodies are already widely tested, we can foresee early clinical investigation.

Allen's group carried out an interesting comparison of anti-CD 19 liposomal doxorubicin when the whole monoclonal antibody or the Fab' or the single chain fragment were bounded on the surface of liposomes [125].

To choose the best candidate for a further clinical application, a clear understanding of the advantages and disadvantages on the use of one type over the other as targeting ligand is extremely important during the preclinical phases in term of efficacy, safety, applicability and costs. Binding properties of mAb- and Fab-targeted liposomes were found to be similar and improved compared to non-targeted liposomes. However, scFv-immunoliposomes exhibited a decreased affinity which was predictable when considering their size rendering the small fragment ease to be masked by PEG chains. The Fc-mediated clearance was circumvented when Fab fragments were used and their blood levels were comparable to classic long-circulating liposomes. ScFv-liposomes showed a lower circulation lifetime, but this was likely due to the presence of Poly-His or c-myc tags necessary to trace and quantify the single chain fragment during analysis, but 
normally absent during clinical uses. Allen's team concluded the study considering scFv-immunoliposomes easy to fabricate, with a more economical production compared to $\mathrm{mAb}$ and the absence of tags in clinical application needs to be considered to reduce their liver uptake and possible adverse effects in humans.

Many other antibodies have been exploited for an active targeting, few examples are GD2-targeted immunoliposomes loaded with fenretinide for the treatment of neuroblastoma [77], or immunoliposomes directed against the accessible domain (KDR) of the vascular endothelial growth factor receptor with the aim of delivering antiangiogenic drugs [149]. One more recent study concerning anti-CD22 immunoliposomes loaded with doxorubicin showed an improved efficacy against B-cell lymphoma in vitro and in vivo. Anti$\mathrm{CD} 22$ is a new therapeutic antibody whose clinical trials are now on going [150].

The most important concept to retain from this section was highlighted by Kirpotin et al. in 2006 [151]. They claimed and proved through experimental studies that the fundamental difference between the second and the third generation of liposomes is not in the quantity of nanocarriers accumulated into tumor tissue, but rather in the quality of their accumulation. Indeed, targeted-liposomes do not accumulate in a major amount compared to sterically stabilized liposomes, a thought widely diffused during the early stages of immunoliposomes development, but once in the tumor, immunoliposomes are able to penetrate within cancer cells more efficiently compared to non-targeted liposomes. This discovery can then explain the improved antitumor activity often found in the case of active targeted liposomes.

Immuno-targeting is probably the most promising approach, because the design of liposome linked to an antibody, such as trastuzumab, and containing a powerful anticancer agent may constitute a double shot gun. In such a case, the antibody may block the cell proliferative and/or disseminative effects of the recognized antigen receptor, favoring endocytosis of the nanosystem and the delivery of the encapsulated anticancer agent within the cell complete antineoplastic effect. It is also likely, as suggested by works dealing with various receptor targeted polymers, dendrimers or liposomes, that the active targeting of nanocarriers minimize the influence of the architecture, composition, size and molecular mass of nanocarriers on the efficacy of the treatment [152].

\subsection{Lipoplexes}

In liposome domain, when we describe the latest developments and improvements, we are obliged to mention the delivery of nucleic acids, peptides or siRNA through a nonviral approach consisting in the use of cationic lipids which gives rise to a new class of liposomes usually called lipoplexes.

In this review only the main and basic concepts will be cited; the complexity and variety of structures proposed nowadays, as well as the different pharmacokinetics and biodistribution behaviour compared to classical liposomes worth the editing of a entire specific review [153], hence only the most important notions will be hereinafter described.
In the case of DNA, proteins and peptides, unable to spontaneously cross cell membranes, an active transport mechanism is fundamental to explain their functions. Viral vectors for DNA delivery suffer from non-specificity and risk of virus-induced immune responses, thus a new field of investigation was opened to find non-viral vectors able to delivery macromolecules.

The addition of positive charges to nanodevices can enhance their uptake by cells and the use of cationic lipids as transfection vectors for intracellular DNA delivery is a concept suggested more than 20 years ago [85]. The structure of lipoplexes is based on the strong electrostatic interactions between the positive charges of lipids and the negative charges of phosphate groups present on DNA [154]. Moreover, positive charges facilitate their interaction with negatively charged cells and promote particle internalization. Fig. (3) shows the possible structure conceived for this new class of lipid nanoparticles. Endocytosis seems to be the most common internalization process for lipoplexes [155], and interestingly DNA does not end in lysosomes but it is normally released into cytoplasm thank to positive charged lipids which destabilize endosome membranes and promote DNA cytoplasm liberation [85].

Liposome-based gene therapy approaches, including those directed against drug resistance mechanisms, have been extensively described by Pedroso de Lima and coworkers $[156,157]$. Ligand-targeted lipoplex are now developing, for example lipoplexes targeting the CD44 hyaluronic acid receptor showed efficient transfection of breast cancer cell lines [158]. Liposomes are also used to target antisense oligonucleotides. Antisense nucleotides against MDR1 gene were as well conceived already in the early 1990's by Thierry et al. [159]. Coated cationic liposomes made of a central core of cationic phospholipids bound to oligonucleotides and surrounded by an external shell of neutral lipids showed an improved efficacy against neuroblastoma in vitro and in vivo [160].

Octamer of arginin attached to the surface of siRNA loaded liposomes showed an enhanced intracellular delivery and silencing of the targeted gene [161].

Similarly, hyaluronic acid-modified liposomes for the targeted delivery of anti-telomerase siRNA to CD44expressing lung cancer cells showed and improved transfection efficacy [162].

As we can see many different approaches are now developing over this new field of lipid nanocarrier and the first results encourage researchers to investigate further more.

\section{FROM THE BENCH TO THE BEDSIDE: NEW CLINICAL TRIALS AND LIPOSOMES COMMER- CIALLY AVAILABLE}

After more than 25 years of preclinical and clinical investigation on liposome domain, the first commercially available liposomal formulation for the treatment of cancer was obtained in the late 1990's. The high number of variable factors related to lipid systems and their preparation methods sometimes not easily translatable to industrial production, slowed down the way to reach market place. 
Table (2) summarizes the anticancer drugs approved by FDA when administered as liposomal formulation. This section will describe the main characteristics of each formulation and later on, the main ongoing clinical trials over new anticancer drugs encapsulated into liposomes (table 3).

\subsection{Anticancer Drugs Loaded Liposome: FDA-Approved and Commercially Available}

In 1995, Stealth ${ }^{\circledR}$ liposomes containing doxorubicin have been approved by FDA for the treatment of Kaposi's sarcoma. Doxil $^{\mathbb{B}}$ (for the US market) or Caelyx ${ }^{\mathbb{B}}$ (for European market) is composed of a lipid barrier made of DSPC:CHOL:DSPE-PEG and doxorubicin is loaded through the ammonium sulfate preparation method taking advantages from the ionizable properties of the active molecule. Its use has been extended to ovarian cancer in 1999 and breast cancer the same year in the United States and in 2003 in Europe and Canada [163]. Doxil ${ }^{\mathbb{B}}$ received a large success over patients tolerability and for this reason, it is now under clinical investigation for many combinatory anticancer therapies.

Another well known doxorubicin loaded liposome is the Myocet ${ }^{\circledR}$ formulation. It has been approved in Europe, in 2000 , for the treatment of metastatic breast cancer in combination with cyclophosphamide, but it is still under clinical investigation in the United States. Such liposomes are made by ePC and cholesterol without any polymer bounded on the surface able to render these liposomes sterically stabilized (PEG or GM1).

Daunorubicin is another anticancer drug widely investigated for liposomal formulations. In 1995, DaunoXome ${ }^{\circledR}$ was approved by FDA and entered on the market one year later for the treatment of Kaposi's sarcoma. DaunoXome ${ }^{\circledR}$ contains an aqueous solution of the citrate salt of daunorubicin encapsulated within lipid vesicles composed of a lipid bilayer of DSPC and cholesterol (2:1 molar ratio), with a mean diameter of about $45 \mathrm{~nm}$. Daunorubicin loaded liposomes are now in clinical trials for the treatment of other types of cancer as well as in combinatory therapies.

DepoCyt $^{\circledR}$ (cytarabine liposome injection) reached the market in 1999. It is a sustained-release formulation of the active cytarabine designed for direct administration into the cerebrospinal fluid. Approved for the treatment of neoplastic meningitis, it is made of multilamellar vesicles composed of cholesterol, $4.1 \mathrm{mg} / \mathrm{mL}$; dioleoylphosphatidylcholine (DOPC), $5.7 \mathrm{mg} / \mathrm{mL}$; and dipalmitoylphosphatidylglycerol (DPPG), $1.0 \mathrm{mg} / \mathrm{mL}$ and containing $10 \mathrm{mg} / \mathrm{mL}$ of cytarabine.

Vincristine sulfate liposomes (Marqibo ${ }^{\circledR}$ ) designed by Hana Biosciences failed to enter into the market in 2005 when US FDA voted unanimously against recommending accelerated approval for Marqibo ${ }^{\circledR}$ as a treatment for patients with relapsed aggressive non-Hodgkin's lymphoma. In 2009 phase II clinical trials were completed for pediatric and adolescent patients with relapsed malignancies and recently, in 2012, it received the FDA agreement for the treatment of Acute Lymphoblastic Leukaemia (ALL).

\subsection{Ongoing Clinical Trials}

Section 4.1. describes the few anticancer liposomal drugs available on commerce, but visiting the website http://www.clinicaltrials.gov/, a large number of ongoing clinical trials reveals the use of different liposome formulations.

\section{2.a. Late Stages in Clinical Trials}

This section will be dedicated to the most advanced stages of liposome clinical trials and the most innovative ones (Table 3).

The Memorial Sloan-Kettering Cancer Center of New York completed phase II on liposomal paclitaxel (PNU93914) in treating patients with locally advanced or metastatic cancer of the oesophagus.

Marqibo ${ }^{\circledR}$, already approved for ALL, completed phase II clinical trial for the treatment of pediatric and adolescent patients with relapsed malignancies such as soft tissue sarcoma, lymphoma, leukaemia, Wilms' tumor, osteosarcoma and is now taking part of a phase II study in patients with metastatic uveal melanoma.

A phase II clinical trial has been completed in 2008 to determine the tumor concentration of liposomal 9-nitro20(S)-camptothecin (L9NC) administered by inhalation and to study the overall response rate in patients with non-smallcell lung cancer. A pharmacology study of L9NC in the plasma, and lungs after aerosolization has been performed as well and more recent phase II clinical trial has been completed in 2009 to study the association of L9NC and temozolomide for the treatment of Ewing's sarcoma.

A phase II clinical trial has been completed in March 2010 for lurtotecan liposome in treating patients with ovarian epithelial cancer and patients with metastatic or locally recurrent head and neck cancer. Randomized phase II trial has been carried out to compare the effectiveness of two treatment regimens of lurtotecan liposome in patients who have advanced or recurrent ovarian epithelial cancer, primary fallopian tube cancer, or peritoneal cancer that has been previously treated with chemotherapy. Liposomal SN-38 in treating patients with metastatic colorectal cancer is now in phase II clinical study. Encapsulated SN38 (LE-SN38) is an oncology drug product consisting of the active metabolite of irinotecan (CPT-11). Liposomal formulation of a relatively insoluble compound (SN38) and improvement in drug delivery (pharmacodynamic profile) may be obtained. An improved safety and efficacy profile, compared with the prodrug CPT-11 is expected as well. This rationale is supported by the results from animal toxicity studies in preclinical trials both the mouse and dog and during phase I clinical trial.

Neopharm just completed phase I clinical study on liposome encapsulating mitoxantrone (LEM) in patients with advanced malignancies. Liposome entrapped mitoxantrone (LEM) is a mixture of commercially available mitoxantrone $\mathrm{HCl}\left(\right.$ Novantrone ${ }^{\circledR}$ ) and a combination of lyophilized lipids. Mitoxantrone, the active agent in the investigational formulation, is a currently marketed chemotherapeutic agent.

Many other studies are mentioned in the official website of clinical trials http://www.clinicaltrials.gov/; here authors wanted to share some of the latest updates on liposome research moving from the bench to the bedside. 
Table 2. Commercially Available Liposomal Formulations for Cancer Therapy

\begin{tabular}{|c|c|c|c|}
\hline Name & Drug & Year & Cancer Type \\
\hline \hline \multirow{2}{*}{ Doxil $^{\circledR}-$ Caelyx $^{\circledR}$} & & 1995 & Kaposi's sarcoma \\
& doxorubicin & 1999 & Ovarian cancer (USA) \\
\cline { 2 - 4 } & & 2003 & Ovarian cancer (Canada, EU) \\
\hline Myocet $^{\circledR}$ & doxorubicin & 2000 & Metastatic breast cancer \\
\hline DaunoXome $^{\circledR}$ & daunorubicin & 1996 & Kaposi's sarcoma \\
\hline DepoCyte $^{\circledR}$ & cytarabine & 1999 & Neoplastic meningitis \\
\hline Marqibo $^{\circledR}$ & vincristine & 2012 & Acute lymphoblastic leukaemia \\
\hline
\end{tabular}

Table 3. Ongoing Clinical Trials

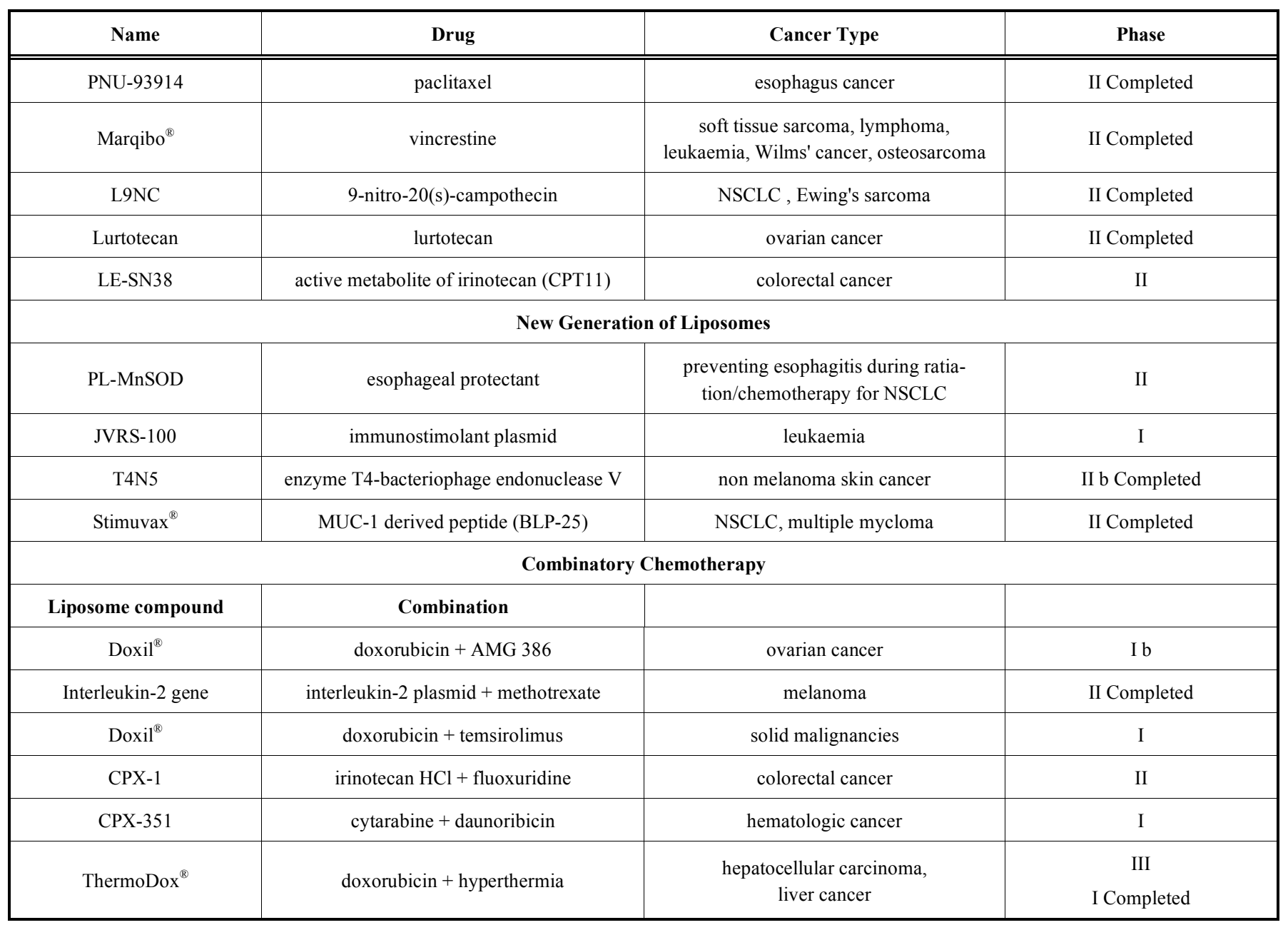

\section{2.b. Clinical Trials on the Last Generation of Liposomes}

Regarding the last generation of liposome containing DNA (lipoplexes), the oesophageal protectant (MnSOD) used to prevent esophagitis during radiation/chemotherapy treatment for non-small cell lung cancer (NSCLC) need to be mentioned. A phase I-II study was carried out to evaluate the feasibility, safety, and efficacy of swallowed MnSOD plas$\mathrm{mid} /$ liposome $(\mathrm{PL})$ transgene given as protection against radiation-induced esophagitis during concurrent paclitaxel and carboplatin chemotherapy with thoracic radiation in subjects with locally advanced NSCLC. This study is currently suspended for interim data analysis.

Merck KGaA completed in August 2012 two phase IIb studies of BLP25 liposome vaccine for immunotherapy of non-small cell lung cancer $\left(\right.$ Stimuvax ${ }^{\circledR}$ ) and patients with slowly progressive multiple myeloma with no symptoms and 
who have had no chemotherapy. BLP25 is a liposomeencapsulated peptide vaccine consisting of a synthetic peptide derived from the mucin-1 (MUC-1) antigen with potential antineoplastic activity. Upon vaccination, MUC-1 peptide vaccine may stimulate the host immune system to mount a cytotoxic T lymphocyte (CTL) response against MUC-1expressing tumor cells, resulting in growth inhibition. Merck is now recruiting patients for a phase II study for the treatment of rectal, colorectal and prostate cancer.

A phase IIb clinical trial has been completed for T4N5 liposomal lotion in preventing the recurrence of nonmelanoma skin cancer in patients who have undergone a kidney transplant. T4N5 is a topical lotion that contains the enzyme $\mathrm{T} 4-$ bacteriophage endonuclease $\mathrm{V}$. In vitro and in vivo studies indicate that T4N5 liposomes increases repair of DNA damage caused by UV irradiation. T4N5 liposome lotion is also in phase III for the treatment of patients with Xeroderma pigmentosum who develop skin lesions in sun-affected areas. These skin lesions, such as actinic keratoses, can develop into skin cancer. Such lotion may reduce actinic keratoses or other sun-induced skin damage in patients with Xeroderma pigmentosum.

The lipid/DNA complexes (JVRS-100) are now in phase I clinical trial for the treatment of patients with relapsed or refractory leukaemia. JVRS-100 is a cationic liposome DNA complex (CLDC) composed of cationic DOTIM/cholesterol liposomes and plasmid DNA. The addition of peptide or protein antigens to DOTIM/cholesterol has been shown to produce a potent adjuvant effect following vaccination, with induction of enhanced CD4+ and CD8+ T-cell and antibody. JVRS-100 exhibits marked immunostimulatory properties, particularly for the induction of T-dependent antibody, characteristic of $\mathrm{T}$ helper 1 (Th1) responses (IFN-predominant cytokine secretion pattern) and CD8+ CTL responses [164].

Phase I clinical study has been completed in February 2012 for DOTAP:cholesterol-Fus1 in non-small-cell lung cancer. This study is an attempt to transfer gene fus 1 into cancer cells, using the formulation DOTAP:cholesterolfus1. Researchers will also study the highest safe dose and the side-effects of this experimental gene transfer at different doses. Tests to see if there are any effects on tumor size on both non-small cell lung cancer and small cell lung cancer (SCLC) will be carried out as well.

\section{2.c. Combinatory Chemotherapy}

New strategies in chemotherapy are now developing forward combination of different anticancer drugs in order to take advantage from their synergism, possibly diminish doses and consequent side-effects and finally combat the disease aiming different targets.

The same direction has been taken by liposomes as well. Liposomal anticancer drugs whose efficacy is already well established (i.e. liposomal doxorubicin) are now under clinical studies to confirm their combination with various active molecules.

Few examples are the phase II trial of pegylated liposomal doxorubicin in combination with docetaxel with or without trastuzumab for the treatment of metastatic breast cancer [165].
A phase Ib study of AMG 386 in combination with pegylated liposomal doxorubicin in subjects with advanced recurrent epithelial ovarian cancer is ongoing. AMG 386 is a selective angiopoietin 1/2-neutralizing peptibody that inhibits angiogenesis by preventing interaction between angiopoietins and Tie 2 receptors. It is hypothesized that $\mathrm{AMG}$ 386 , in combination with pegylated liposomal doxorubicin will be safe and well tolerated in subjects with recurrent ovarian cancer.

The injection of Interleukin-2 plasmid lipoplex combined with methotrexate for the treatment of head and neck cancer completed a phase II clinical trial recently.

A phase I study of Doxil ${ }^{\circledR}$ and temsirolimus in resistant solid malignancies is now under investigation. The Mammalian Target of Rapamycin (mTOR) is a large polypeptide serine/threonine kinase important as regulator of cancer cell cycle, proliferation, invasion, and angiogenesis. Inhibition of mTOR would result in arrest of cell growth in G1 phase of the cell cycle. Temsirolimus is a soluble ester analogue of rapamycin (sirolimus) which has shown impressive cytostatic effects on a wide variety of cancer such as T-cell leukaemia, glioblastoma, melanoma, prostate, breast, renal cell, and pancreatic cells in animal models. In phase I trials, temsirolimus has been investigated as a single agent and evidence of activity was observed in patients with both breast and renal cancer. Major tumor responses were noted in phase I trials in patients previously treated with lung, breast, renal as well as neuroendocrine tumors. Minor responses were noted in soft tissue sarcoma, endometrial, and cervical carcinoma. The study has the aim of determining any anti-tumor activity and response from the combination of temsirolimus and pegylated liposomal doxorubicin in treatment of patients with resistant solid malignancies.

Even more interesting clinical trials from liposome technical point of view are the combination of different antitumor drugs in the same liposomal formulation. A phase II clinical study is carried out by Celator Pharmaceuticals over CPX-1 (irinotecan $\mathrm{HCl}$ : floxuridine) liposome injection in patients with advanced colorectal cancer. CPX-1 is a liposomal formulation of a fixed combination of antineoplastic drugs irinotecan $\mathrm{HCl}$ and floxuridine. The two drugs are present inside the liposome in a fixed 1:1 molar ratio and CPX-1 was developed as a means of delivering and preserving a fixed 1:1 molar ratio of the two drugs. This ratio was found, in vitro and in vivo cancer models, to have synergistic anticancer activity. Preservation and delivery of this ratio is important because other ratios of these two drugs have been found to be antagonistic or only additive. Both floxuridine and irinotecan $\mathrm{HCl}$ are active chemotherapeutic agents, each approved for clinical use in the United States and Canada for colorectal cancer. Current practice routinely administers 5fluorouracil with irinotecan in combination regimens in first or second line treatment without the means of preserving the synergistic ratio.

Celator Pharmaceuticals has also completed a phase I study over CPX-351 (cytarabine : daunorubicin) liposome injection in patients with advanced haematological cancer. CPX-351 is a liposomal formulation of a fixed combination of the antineoplastic drugs cytarabine and daunorubicin present inside the liposome in a $5: 1$ molar ratio. The 
development of CPX-351 was based on: i) defining a synergistic ratio of the two active moieties, cytarabine and daunorubicin, using cell-based screening assays and, ii) designing a liposomal drug carrier to maintain this ratio after intravenous administration. CPX-351 was found to be more active in vivo models of cancer than combinations of conventional cytarabine and daunorubicin. The primary objective of the phase I study was to determine the recommended dose of CPX-351 for use in a phase II efficacy study in patients with leukaemia. Preliminary evidence of antitumor activity has been determined as well.

A combination of hyperthermia, temperature sensitive liposomes and radiofrequency ablation is now under investigation for various neoplastic pathologies. Celsion completed phase III study to determine whether ThermoDox ${ }^{\mathrm{TM}}$, a thermally sensitive liposomal doxorubicin, is effective in the treatment of non-resectable hepatocellular carcinoma when used in conjunction with radiofrequency ablation. A phase I clinical trial has been already completed in March 2010 studying the best dose of liposomal doxorubicin when given with radiofrequency ablation in treating patients with primary or metastatic liver tumours. Celsion is moreover recruiting patients with colon cancer and liver metastasis to undergo a new phase II clinical trial.

This section has been dedicated to the latest developments in term of liposomes currently under investigation in clinical therapy. A modest overview has been given to understand how large is the fan of liposome applications and only few studies are here described, however more than three hundred trails are ongoing or just completed for the treatment of cancers using liposomal anticancer drugs.

\section{CONCLUSION}

In the past decade, pharmaceutical domain emphasized its researches over drug delivery systems in order to modify drugs behaviour according to the treated disease and the variability of responses from one patient to another.

Liposomes are undoubtedly one of the most investigated colloidal systems in the field of nanomedicine. As described in research studies mentioned before, liposomes can be used as a means to modify pharmacokinetics and biodistribution of anticancer drugs [17]. Molecules presenting a fast metabolism in vivo can be protected by the lipid shell in the blood stream, and can accumulate in the desired tissue maintaining their antitumor efficacy (Renoir's group unpublished work). Moreover, they can be used as simple vehicle for hydrophobic drugs to permit their intravenous administration [166]. Their controlled release properties are often exploited to bombard continuously and for longer periods of time the tumor tissue in order to obtain a possible overwhelming of drug transporters responsible of drug efflux [17].

Many anticancer molecules such as doxorubicin improved their therapeutic index thank to the encapsulation in liposomal systems [163]. The ability of accumulating in tumors diminishing their interaction with healthy tissues can prevent some undesired side effects likely cardiotoxicity in the case of doxorubicin.

However, one of the most interesting advantages proposed by liposomes is the possibility of combining on the same vector different technologies and approaches to combat the desired pathology. Multifunctional approach involves combination of stimuli-sensitive functions such as hyperthermia with pharmaceutical techniques like long-circulating liposomes modified to become ligand-targeted nanosystems. Magneto-liposomes can be loaded with a $\mathrm{pH}$-gradient technique to introduce ionizable anticancer drugs and $\mathrm{scFv}$ bounded on the surface of liposomes could enhance internalization within tumor cells. Vectors can then be shaped following the intrinsic characteristics of the pathologic area to obtain a specific medication for a specific disease. The use of drug combination is already a common strategy used in clinical therapy, however drug interactions are hard to foreseen when we translate from in vitro to in vivo experiments. Encapsulation of two different drugs in the same liposomal nanocarrier can synchronize the distribution of both molecules if the coencapsulation results to be stable [167]. Mayer and Wang studied the liposomal combination of cisplatin/daunorubicin or cytarabine/daunorubicin as well as doxorubicin/verapamil whose synergism was found to be active on MDR cells [168, 169].

The wide range of studies cited in this review drives us to think that pharmaceutical improvements, from one hand, and always new biological discoveries, on the other hand, can be associated to design the optimized liposomal drug thanks to the possibility of grouping different aspects on the same medication, a distinctive feature difficult to achieve using classical chemotherapy.

Liposomal drugs have been suggested to be the long awaited "magic bullet" for cancer therapy, thanks to their specific accumulation in tumor tissues [170]. However, not all cancers and patients respond to the magic bullet equivalently [171] and some cases of toxic reaction and intolerance have been established [17].

Liposome accumulation in healthy tissues is normally lower compared to free drug, thus the toxicity profile of the active molecule is often modified. Side effects such as nausea, alopecia and vomiting are found to be rare in patients treated with Doxil ${ }^{\mathbb{B}}$ [172]. Liposomal drugs result to be more tolerated, preventing patients to leave treatments because of unbearable toxicities [17]; however, some cases of toxicity must to be taken into account.

In the case of Doxil ${ }^{\circledR}$, the most important side effect consists in dermal lesions (Hand and Foot syndrome; H-F) already described for long continuous infusions of doxorubicin and not observed for bolus injections, thus dosage and treatment schedule need to be adjusted to minimize this toxicity. The official clinical trials website (http://www. clinicaltrials.gov/) mentions a phase III clinical study for the association of an antiperspirant cream (F511) preventing palmarplantar erythrodysesthesia in patients receiving doxorubicin hydrochloride liposome for metastatic breast cancer.

At high doses, liposomes resulted to cause impairment of RES function, hepatomegaly and splenomegaly which has not dramatic consequences in normal patients, but could become a serious concern for immunocompromised patients where the RES organs are the first-line defence against bacterial infections, thus adjusted doses need to be considered when we are in presence of this category of patients. 
Furthermore, little attention has been paid to potential immunogenicity of injectable nanosystems [173]. Pharmacokinetics and therapeutic improvements have been widely described, but immunologic responses have been often neglected. Most of preclinical studies are carried out on immunodepressed animals in order to develop tumor, thus no activation of immune system can be observed. Indeed, immunogenicity can have severe clinical implications when repeated administrations are foreseen, therefore further researches need to be pursued to increase the safety of these systems $[173,174]$.

Unfortunately there is one more limitation for the development of nanocarriers which lies in the translation from the bench to industrial production. Indeed, production of nanocarriers in large amounts encounters serious engineering problems which must be solved before getting access to clinical applications. In addition, the price and cost of immunoliposomes has to be taken into consideration and it constitutes also a serious limitation for their development.

As we can see from these last observations the "panacea" for the treatment of cancer is not yet around the corner. Liposomes have undoubtedly improved anticancer drug therapeutic profiles and the opportunity to develop multi functional nanocarriers promises to alleviate many of the issues still present in clinical cancer therapy.

\section{CONFLICT OF INTEREST}

The author(s) confirm that this article content has no conflicts of interest.

\section{ACKNOWLEDGEMENTS}

We deeply thank Prof. Alain Gouyette for critical discussions and reading of the manuscript. This work was supported by the Ligue Nationale contre le Cancer (Cher, Indre and Hauts-de-Seine committees, grants to J-M. R. and fellowship to G.U.).

\section{ABBREVIATIONS}

$\begin{array}{ll}\text { MLV } & =\text { Multi Lamellar Vesicles } \\ \text { SUV } & =\text { Small Unilamellar Vesicles } \\ \text { IUV } & =\text { Intermediate Size Unilamellar Vesicles } \\ \text { LUV } & =\text { Large Unilamellar Vesicles } \\ \text { REV } & =\text { Reverse Phase Evaporation } \\ \text { ePC } & =\text { Egg-Phosphatidylcholine } \\ \text { Chol } & =\text { Cholesterol } \\ \text { PC } & =\text { Phosphatidylcholine } \\ \text { PG } & =\text { Phosphatidylglicerol } \\ \text { DSPC } & =\text { Distearoyl-Phosphocholine } \\ \text { HSPC } & =\text { Hydrogenated Soybean Phosphatidylcho- } \\ \text { DOPC } & =\text { Dioleoylphosphocholine } \\ \text { DPPC } & =\text { Dipalmitoylphosphocholine } \\ \text { DSPG } & =\text { Distearoyl-Phosphoglycerol }\end{array}$

\begin{tabular}{|c|c|c|}
\hline DPPG & $=$ & Dipalmitoylphosphoglycerol \\
\hline PEG & $=$ & Polyethylene Glycol \\
\hline PEG-PE & $=$ & Polyethylene Glycol Phosphoethanolamine \\
\hline GM1 & $=$ & Monosialoganglioside \\
\hline HDL & $=$ & High Density Lipoproteins \\
\hline C3bn & $=$ & Cleaved Complement Fragment \\
\hline CR1 & $=$ & Complement Receptor 1 \\
\hline ara-C & $=$ & Cyttarabine \\
\hline L-NDDP & $=$ & $\begin{array}{l}\text { Cis-Bis-Neodecanoato-Trans-R,R-1,2-Dia } \\
\text { minocyclohexane Platinum }\end{array}$ \\
\hline$t^{1} / 2$ & $=$ & Half-Life \\
\hline $\mathrm{Tm}$ & $=$ & Transition Phase Temperature \\
\hline EPR & $=$ & Enhanced Permeability Retention \\
\hline RES & $=$ & Reticuloendothelial System \\
\hline VIP & $=$ & Vasoactive Instestinal Peptide \\
\hline RGD & $=$ & Arginine-Glycine-Aspartate Peptides \\
\hline GAG & $=$ & Glycosaminoglycan \\
\hline TAT & $=$ & $\begin{array}{l}\text { Trans-Activating Transcriptional Activator } \\
\text { Protein }\end{array}$ \\
\hline MFL & $=$ & Magnetic-Fluid-Loaded Liposomes \\
\hline mTOR & $=$ & Mammalian Target of Rapamycin \\
\hline T4N5 & $=$ & Enzyme T4-Bacteriophage Endonuclease V \\
\hline L9NC & $=$ & 9-Nitro-20(S)-Campothecin \\
\hline NSCLC & $=$ & Non-Small Cells Lung Cancer \\
\hline SCLC & $=$ & Small Cells Lung Cancer \\
\hline ALL & $=$ & Acute Lymphoblastic Leukaemia \\
\hline
\end{tabular}

\section{REFERENCES}

[1] Johnson, S.M. Bangham, A.D. Potassium permeability of single compartment liposomes with and without valinomycin. Biochim. Biophys. Acta, 1969, 193(1), 82-91.

[2] Johnson, S.M. Bangham, A.D. The action of anaesthetics on phospholipid membranes. Biochim Biophys Acta, 1969, 193(1), 92-104.

[3] Bangham, A.D.; Standish, M.M. Watkins, J.C. Diffusion of univalent ions across the lamellae of swollen phospholipids. J. Mol. Biol., 1965, 13(1), 238-252.

[4] Viriyaroj, A.; Ngawhirunpat, T.; Sukma, M.; Akkaramongkolporn, P.; Ruktanonchai, U. Physicochemical properties and antioxidant activity of gamma-oryzanol-loaded liposome formulations for topical use. Pharm. Dev. Technol., 2009, 14(6), 665-671.

[5] Allen, T.; Stuart, D. Liposomes: Rational design; Marcel Dekker, Inc.: New York, 1999.

[6] Gregoriadis, G. Liposome technology; 2nd; CRC: Boca Raton, FL, 1993.

[7] Maestrelli, F.; Capasso, G.; Gonzalez-Rodriguez, M.L.; Rabasco, A.M.; Ghelardini, C. Effect of preparation technique on the properties and in vivo efficacy of benzocaine-loaded ethosomes. J. Liposome Res., 2009, 19(4), 253-260.

[8] Sivan, S.; Schroeder, A.; Verberne, G.; Merkher, Y.; Diminsky, D. Liposomes act as effective biolubricants for friction reduction in human synovial joints. Langmuir, 2010, 26(2), 1107-1116.

[9] Zaru, M.; Manca, M.L.; Fadda, A.M. Antimisiaris, S.G. Chitosancoated liposomes for delivery to lungs by nebulisation. Colloids Surf B. Biointerfaces, 2009, 71(1), 88-95.

[10] Gulati, M.M.G.; Singh, S.; Singh, M. Lipophilic drug derivatives in liposomes. Int. J. Pharm., 1998, 165, 129-168. 
[11] Betageri, G.V.; Dipali, S.R. Partitioning and thermodynamics of dipyridamole in the n-octanol/buffer and liposome systems. $J$. Pharm. Pharmacol., 1993, 45(10), 931-933.

[12] Tokunaga, Y.; Iwasa, T.; Fujisaki, J.; Sawai, S. Kagayama, A. Liposomal sustained-release delivery systems for intravenous injection. I. Physicochemical and biological properties of newly synthesized lipophilic derivatives of mitomycin C. Chem. Pharm. Bull. (Tokyo), 1988, 36(8), 3060-3069.

[13] Hashida, M.; Sato, K.; Takakura, Y. Sezaki, H. Characterization of a lipophilic prodrug of 5-fluorouracil with a cholesterol promoiety and its application to liposomes. Chem. Pharm. Bull. (Tokyo), 1988, 36(8), 3186-3189.

[14] Hashimoto, K.; Loader, J.E.; Knight, M.S. Kinsky, S.C. Inhibition of cell proliferation and dihydrofolate reductase by liposomes containing methotrexate-dimyristoylphosphatidylethanolamine derivatives and by the glycerophosphorylethanolamine analogs. Biochim. Biophys. Acta, 1985, 816(1), 169-178.

[15] Perez-Soler, R.; Yang, L.Y.; Drewinko, B.; Lauterzstain, J. Khokhar, A.R. Increased cytotoxicity and reversal of resistance to cisdiamminedichloro-platinum(II) with entrapment of cis-Bisneodecanoato-trans-R,R-1,2-diaminocyclohexaneplatinum (II) in multilamellar lipid vesicles. Cancer Res., 1988, 48(16), 4509-4512.

[16] US National Institutes of Health. Specialized information Services: Clinical Trials. http://clinicaltrials.gov/ct2/results?term=LNDDP+liposome (Accessed April 12, 2010)

[17] Drummond, D.C.; Meyer, O.; Hong, K.; Kirpotin, D.B. Papahadjopoulos, D. Optimizing liposomes for delivery of chemotherapeutic agents to solid tumors. Pharmacol. Rev., 1999, 51(4), 691-743.

[18] Katare, O.P.; Vyas, S.P. Dixit, V.K. Effervescent granule based proliposomes of ibuprofen. J. Microencapsul., 1990, 7(4), 455-462.

[19] Garelli, N.; Vierling, P. Incorporation of new amphiphilic perfluoroalkylated bipyridine platinum and palladium complexes into liposomes: stability and structure-incorporation relationships. Biochim. Biophys. Acta, 1992, 1127(1), 41-48.

[20] Kikuchi, H.A.C.; Yachi, K.S.; Hirota Possibility of heat sterilization of liposomes. Chem. Pharm. Bull., 1991, 39, 1018-1022.

[21] Zuidam, N.J.; Lee, S.S.; Crommelin, D.J. Sterilization of liposomes by heat treatment. Pharm Res., 1993, 10(11), 1591-1596.

[22] Gabizon, A.A.; Barenholz, Y. Bialer, M. Prolongation of the circulation time of doxorubicin encapsulated in liposomes containing a polyethylene glycol-derivatized phospholipid: pharmacokinetic studies in rodents and dogs. Pharm. Res., 1993, 10(5), 703-708.

[23] Bally, M.B.; Nayar, R.; Masin, D.; Hope, M.J.; Cullis, P.R. Liposomes with entrapped doxorubicin exhibit extended blood residence times. Biochim. Biophys. Acta, 1990, 1023(1), 133-139.

[24] Gabizon, A. Tailoring liposomes for cancer drug delivery: from the bench to the clinic. Ann. Biol. Clin., (Paris), 1993, 51(9), 811-813.

[25] Swasono, R.T.; Mouri, R.; Morsy, N.; Matsumori, N.; Oishi, T. Sterol effect on interaction between amphidinol 3 and liposomal membrane as evidenced by surface plasmon resonance. Bioorg. Med. Chem. Lett., 2010, 20(7), 2215-2218.

[26] Mayhew, E.; Rustum, Y.M.; Szoka, F. Papahadjopoulos, D. Role of cholesterol in enhancing the antitumor activity of cytosine arabinoside entrapped in liposomes. Cancer Treat. Rep., 1979, 63(1112), 1923-1928.

[27] Abraham, S.A.; Edwards, K.; Karlsson, G.; Hudon, N.; Mayer, L.D. An evaluation of transmembrane ion gradient-mediated encapsulation of topotecan within liposomes. J. Control Release, 2004, 96(3), 449-461.

[28] Celano, M.; Calvagno, M.G.; Bulotta, S.; Paolino, D.; Arturi, F. Cytotoxic effects of gemcitabine-loaded liposomes in human anaplastic thyroid carcinoma cells. BMC Cancer, 2004, 4(63).

[29] Dos Santos, N.; Cox, K.A.; McKenzie, C.A.; van Baarda, F.; Gallagher, R.C. pH gradient loading of anthracyclines into cholesterolfree liposomes: enhancing drug loading rates through use of ethanol. Biochim. Biophys. Acta, 2004, 1661(1), 47-60.

[30] Lasic, D.D.; Frederik, P.M.; Stuart, M.C.; Barenholz, Y. McIntosh, T.J. Gelation of liposome interior. A novel method for drug encapsulation. FEBS Lett., 1992, 312(2-3), 255-258.

[31] Cullis, P.R.; Hope, M.J.; Bally, M.B.; Madden, T.D.; Mayer, L.D. Influence of $\mathrm{pH}$ gradients on the transbilayer transport of drugs, lipids, peptides and metal ions into large unilamellar vesicles. Biochim. Biophys. Acta, 1997, 1331(2), 187-211.

[32] Frezard, F.; Santaella, C.; Montisci, M.J.; Vierling, P. Riess, J.G. Fluorinated phosphatidylcholine-based liposomes: $\mathrm{H}+\mathrm{Na}+$ perme- ability, active doxorubicin encapsulation and stability, in human serum. Biochim. Biophys. Acta, 1994, 1194(1), 61-68.

[33] Ramsay, E.; Alnajim, J.; Anantha, M.; Zastre, J.; Yan, H. A novel liposomal irinotecan formulation with significant anti-tumour activity: use of the divalent cation ionophore A23187 and coppercontaining liposomes to improve drug retention. Eur. J. Pharm. Biopharm., 2008, 68(3), 607-617.

[34] Clerc, S. Barenholz, Y. Loading of amphipathic weak acids into liposomes in response to transmembrane calcium acetate gradients. Biochim. Biophys. Acta, 1995, 1240(2), 257-265.

[35] Subramanian, D. Muller, M.T. Liposomal encapsulation increases the activity of the topoisomerase I inhibitor topotecan. Oncol. Res., 1995, 7(9), 461-469.

[36] Burke, T.G. Gao, X. Stabilization of topotecan in low $\mathrm{pH}$ liposomes composed of distearoylphosphatidylcholine. J. Pharm. Sci., 1994, 83(7), 967-969.

[37] Embree, L.; Gelmon, K.A.; Lohr, A.; Mayer, L.D.; Coldman, A.J. Chromatographic analysis and pharmacokinetics of liposomeencapsulated doxorubicin in non-small-cell lung cancer patients. $J$. Pharm. Sci., 1993, 82(6), 627-634.

[38] Parr, M.J.; Masin, D.; Cullis, P.R. Bally, M.B. Accumulation of liposomal lipid and encapsulated doxorubicin in murine Lewis lung carcinoma: the lack of beneficial effects by coating liposomes with poly(ethylene glycol). J. Pharmacol. Exp. Ther., 1997, 280(3), 1319-1327.

[39] Senior, J.; Crawley, J.C. Gregoriadis, G. Tissue distribution of liposomes exhibiting long half-lives in the circulation after intravenous injection. Biochim. Biophys. Acta, 1985, 839(1), 1-8.

[40] Gabizon, A. Papahadjopoulos, D. The role of surface charge and hydrophilic groups on liposome clearance in vivo. Biochim Biophys Acta, 1992, 1103(1), 94-100.

[41] Senior, J.H. Fate and behavior of liposomes in vivo: a review of controlling factors. Crit. Rev. Ther. Drug Carrier Syst., 1987, 3(2), 123-193.

[42] Ahl, P.L.; Bhatia, S.K.; Meers, P.; Roberts, P.; Stevens, R. Enhancement of the in vivo circulation lifetime of L-alphadistearoylphosphatidylcholine liposomes: importance of liposomal aggregation versus complement opsonization. Biochim. Biophys. Acta, 1997, 1329(2), 370-382.

[43] Senior, J. Gregoriadis, G. Is half-life of circulating liposomes determined by changes in their permeability? FEBS Lett., 1982, 145(1), 109-114.

[44] Sankaram, M.B. Thompson, T.E. Interaction of cholesterol with various glycerophospholipids and sphingomyelin. Biochemistry, 1990, 29(47), 10670-10675.

[45] Seymour, L.W. Passive tumor targeting of soluble macromolecules and drug conjugates. Crit. Rev. Ther. Drug Carrier Syst., 1992, 9(2), 135-187.

[46] Vaage, J.; Barbera-Guillem, E.; Abra, R.; Huang, A. Working, P. Tissue distribution and therapeutic effect of intravenous free or encapsulated liposomal doxorubicin on human prostate carcinoma xenografts. Cancer, 1994, 73(5), 1478-1484.

[47] Litzinger, D.C.; Buiting, A.M.; van Rooijen, N. Huang, L. Effect of liposome size on the circulation time and intraorgan distribution of amphipathic poly(ethylene glycol)-containing liposomes. Biochim. Biophys. Acta, 1994, 1190(1), 99-107.

[48] Moghimi, S.M. Machanisms of splenic clearance of blood cells and particles: towards development of new splenotropic agents. $\mathrm{Ad}$ vanced drug delivery reviews, 1995, 17, 103-115.

[49] Hobbs, S.K.; Monsky, W.L.; Yuan, F.; Roberts, W.G.; Griffith, L. Regulation of transport pathways in tumor vessels: role of tumor type and microenvironment. Proc. Natl. Acad. Sci. US A, 1998, 95(8), 4607-4612.

[50] Yuan, F.; Leunig, M.; Huang, S.K.; Berk, D.A.; Papahadjopoulos, D. Microvascular permeability and interstitial penetration of sterically stabilized (stealth) liposomes in a human tumor xenograft. Cancer Res., 1994, 54(13), 3352-3356.

[51] Huang, S.K.; Martin, F.J.; Jay, G.; Vogel, J.; Papahadjopoulos, D. Extravasation and transcytosis of liposomes in Kaposi's sarcomalike dermal lesions of transgenic mice bearing the HIV tat gene. Am. J. Pathol., 1993, 143(1), 10-14.

[52] Jain, R.K. Tumor physiology and antibody delivery. Front Radiat. Ther. Oncol., 1990, 24(32-46; discussion 64-38.

[53] Zhang, R.D.; Price, J.E.; Fujimaki, T.; Bucana, C.D. Fidler, I.J. Differential permeability of the blood-brain barrier in experimental 
brain metastases produced by human neoplasms implanted into nude mice. Am. J. Pathol., 1992, 141(5), 1115-1124.

[54] Gabizon, A. Papahadjopoulos, D. Liposome formulations with prolonged circulation time in blood and enhanced uptake by tumors. Proc. Natl. Acad. Sci. U S A, 1988, 85(18), 6949-6953.

[55] Mayer, L.D.; Dougherty, G.; Harasym, T.O. Bally, M.B. The role of tumor-associated macrophages in the delivery of liposomal doxorubicin to solid murine fibrosarcoma tumors. J. Pharmacol. Exp. Ther., 1997, 280(3), 1406-1414.

[56] van Bree, C.; Krooshoop, J.J.; Rietbroek, R.C.; Kipp, J.B. Bakker, P.J. Hyperthermia enhances tumor uptake and antitumor efficacy of thermostable liposomal daunorubicin in a rat solid tumor. Cancer Res., 1996, 56(3), 563-568.

[57] Gaber, M.H.; Hong, K.; Huang, S.K. Papahadjopoulos, D. Thermosensitive sterically stabilized liposomes: formulation and in $v i$ tro studies on mechanism of doxorubicin release by bovine serum and human plasma. Pharm. Res., 1995, 12(10), 1407-1416.

[58] Harris, A.L. Hochhauser, D. Mechanisms of multidrug resistance in cancer treatment. Acta Oncol., 1992, 31(2), 205-213.

[59] Gillet, J.P.; Efferth, T.; Remacle, J. Chemotherapy-induced resistance by ATP-binding cassette transporter genes. Biochim. Biophys. Acta, 2007, 1775(2), 237-262.

[60] Jabr-Milane, L.S.; van Vlerken, L.E.; Yadav, S. Amiji, M.M. Multi-functional nanocarriers to overcome tumor drug resistance. Cancer Treat Rev., 2008, 34(7), 592-602.

[61] Lemos, C.; Jansen, G. Peters, G.J. Drug transporters: recent advances concerning BCRP and tyrosine kinase inhibitors. $B r . J$. Cancer, 2008, 98(5), 857-862.

[62] Kimura, Y.; Morita, S.Y.; Matsuo, M. Ueda, K. Mechanism of multidrug recognition by MDR1/ABCB1. Cancer Sci., 2007, 98(9), 1303-1310.

[63] Paterson, J.K.; Shukla, S.; Black, C.M.; Tachiwada, T.; Garfield, S. Human ABCB6 localizes to both the outer mitochondrial membrane and the plasma membrane. Biochemistry, 2007, 46(33), 9443-9452.

[64] Mamot, C.; Drummond, D.C.; Hong, K.; Kirpotin, D.B. Park, J.W. Liposome-based approaches to overcome anticancer drug resistance. Drug Resist. Updat., 2003, 6(5), 271-279.

[65] Soussi, T. Wiman, K.G. Shaping genetic alterations in human cancer: the p53 mutation paradigm. Cancer Cell, 2007, 12(4), 303-312.

[66] Michalak, E.; Villunger, A.; Erlacher, M. Strasser, A. Death squads enlisted by the tumour suppressor p53. Biochem. Biophys. Res. Commun., 2005, 331(3), 786-798.

[67] Mayer, L.D. Shabbits, J.A. The role for liposomal drug delivery in molecular and pharmacological strategies to overcome multidrug resistance. Cancer Metastasis Rev., 2001, 20(1-2), 87-93.

[68] Goren, D.; Horowitz, A.T.; Tzemach, D.; Tarshish, M.; Zalipsky, S. Nuclear delivery of doxorubicin via folate-targeted liposomes with bypass of multidrug-resistance efflux pump. Clin. Cancer Res., 2000, 6(5), 1949-1957.

[69] Oudard, S.; Thierry, A.; Jorgensen, T.J. Rahman, A. Sensitization of multidrug-resistant colon cancer cells to doxorubicin encapsulated in liposomes. Cancer Chemother. Pharmacol., 1991, 28(4), 259-265.

[70] Mickisch, G.H.; Rahman, A.; Pastan, I. Gottesman, M.M. Increased effectiveness of liposome-encapsulated doxorubicin in multidrugresistant-transgenic mice compared with free doxorubicin. J. Natl. Cancer Inst., 1992, 84(10), 804-805.

[71] Krishna, R.; St-Louis, M. Mayer, L.D. Increased intracellular drug accumulation and complete chemosensitization achieved in multidrug-resistant solid tumors by co-administering valspodar (PSC 833) with sterically stabilized liposomal doxorubicin. Int. J. Cancer, 2000, 85(1), 131-141.

[72] Lee, E.S.; Na, K. Bae, Y.H. Doxorubicin loaded pH-sensitive polymeric micelles for reversal of resistant MCF-7 tumor. J. Control Release, 2005, 103(2), 405-418.

[73] Wu, J.; Lu, Y.; Lee, A.; Pan, X.; Yang, X. Reversal of multidrug resistance by transferrin-conjugated liposomes co-encapsulating doxorubicin and verapamil. J. Pharm. Pharm. Sci., 2007, 10(3), 350-357.

[74] Zalipsky, S.; Saad, M.; Kiwan, R.; Ber, E.; Yu, N. Antitumor activity of new liposomal prodrug of mitomycin $\mathrm{C}$ in multidrug resistant solid tumor: insights of the mechanism of action. J. Drug Target, 2007, 15(7-8), 518-530.
[75] Krieger, M.L.; Eckstein, N.; Schneider, V.; Koch, M.; Royer, H.D. Overcoming cisplatin resistance of ovarian cancer cells by targeted liposomes in vitro. Int. J. Pharm., 2010, 389(1-2), 10-17.

[76] Gaber, M.H. Modulation of doxorubicin resistance in multidrugresistance cells by targeted liposomes combined with hyperthermia. J. Biochem. Mol. Biol. Biophys., 2002, 6(5), 309-314.

[77] Torchilin, V.P. Targeted pharmaceutical nanocarriers for cancer therapy and imaging. AAPS J., 2007, 9(2), E128-147.

[78] Devine, D.V. Marjan, J.M. The role of immunoproteins in the survival of liposomes in the circulation. Crit. Rev. Ther. Drug Carrier Syst., 1997, 14(2), 105-131.

[79] Allen, T.M. Hansen, C. Pharmacokinetics of stealth versus conventional liposomes: effect of dose. Biochim. Biophys. Acta, 1991, 1068(2), 133-141.

[80] Oku, N.; Tokudome, Y.; Asai, T. Tsukada, H. Evaluation of drug targeting strategies and liposomal trafficking. Curr. Pharm. Des., 2000, 6(16), 1669-1691.

[81] Harasym, T.O.; Cullis, P.R. Bally, M.B. Intratumor distribution of doxorubicin following i.v. administration of drug encapsulated in egg phosphatidylcholine/cholesterol liposomes. Cancer Chemother. Pharmacol., 1997, 40(4), 309-317.

[82] Papahadjopoulos, D.; Allen, T.M.; Gabizon, A.; Mayhew, E.; Matthay, K. Sterically stabilized liposomes: improvements in pharmacokinetics and antitumor therapeutic efficacy. Proc. Natl. Acad. Sci. US A, 1991, 88(24), 11460-11464.

[83] Huang, S.K.; Mayhew, E.; Gilani, S.; Lasic, D.D.; Martin, F.J. Pharmacokinetics and therapeutics of sterically stabilized liposomes in mice bearing C-26 colon carcinoma. Cancer Res., 1992, 52(24), 6774-6781.

[84] Napper, D. Polymeric Stabilization of Colloidal Dispersions; Academic Press: New York city, 1983.

[85] Torchilin, V. Multifunctional and stimuli-sensitive pharmaceutical nanocarriers. Eur. J. Pharm. Biopharm., 2009, 71(3), 431-444.

[86] Mori, A.; Klibanov, A.L.; Torchilin, V.P. Huang, L. Influence of the steric barrier activity of amphipathic poly(ethyleneglycol) and ganglioside GM1 on the circulation time of liposomes and on the target binding of immunoliposomes in vivo. FEBS Lett., 1991, 284(2), 263-266.

[87] Allen, T.M.; Austin, G.A.; Chonn, A.; Lin, L. Lee, K.C. Uptake of liposomes by cultured mouse bone marrow macrophages: influence of liposome composition and size. Biochim. Biophys. Acta, 1991, 1061(1), 56-64.

[88] Klibanov, A.L.; Maruyama, K.; Beckerleg, A.M.; Torchilin, V.P. Huang, L. Activity of amphipathic poly(ethylene glycol) 5000 to prolong the circulation time of liposomes depends on the liposome size and is unfavorable for immunoliposome binding to target. Biochim. Biophys. Acta, 1991, 1062(2), 142-148.

[89] Tirosh, O.; Barenholz, Y.; Katzhendler, J. Priev, A. Hydration of polyethylene glycol-grafted liposomes. Biophys. J., 1998, 74(3), 1371-1379.

[90] Woodle, M.C. Surface-modified liposomes: assessment and characterization for increased stability and prolonged blood circulation. Chem. Phys. Lipids, 1993, 64(1-3), 249-262.

[91] Chonn, A.; Semple, S.C. Cullis, P.R. Association of blood proteins with large unilamellar liposomes in vivo. Relation to circulation lifetimes. J. Biol. Chem., 1992, 267(26), 18759-18765.

[92] Allen, T.M. Long-circulating (sterically stabilized) liposomes for targeted drug delivery. Trends Pharmacol. Sci., 1994, 15(7), 215220.

[93] Parr, M.J.; Bally, M.B. Cullis, P.R. The presence of GM1 in liposomes with entrapped doxorubicin does not prevent RES blockade. Biochim. Biophys. Acta, 1993, 1168(2), 249-252.

[94] Price, M.E.; Cornelius, R.M. Brash, J.L. Protein adsorption to polyethylene glycol modified liposomes from fibrinogen solution and from plasma. Biochim. Biophys. Acta, 2001, 1512(2), 191-205.

[95] Moghimi, S.M. Hunter, A.C. Capture of stealth nanoparticles by the body's defences. Crit. Rev. Ther. Drug Carrier Syst., 2001, 18(6), 527-550.

[96] Moghimi, S.M. Humoral-mediated recognition of 'phagocyteresistant' beads by lymph node macrophages of poloxamine-treated rats. Clin. Sci. (Lond), 1998, 95(3), 389-391.

[97] Dams, E.T.; Laverman, P.; Oyen, W.J.; Storm, G.; Scherphof, G.L. Accelerated blood clearance and altered biodistribution of repeated injections of sterically stabilized liposomes. J. Pharmacol. Exp. Ther., 2000, 292(3), 1071-1079. 
[98] Szebeni, J.; Baranyi, L.; Savay, S.; Milosevits, J.; Bunger, R. Role of complement activation in hypersensitivity reactions to doxil and hynic PEG liposomes: experimental and clinical studies. $J$. Liposome Res., 2002, 12(1-2), 165-172.

[99] Moghimi, S.M. Szebeni, J. Stealth liposomes and long-circulating nanoparticles: critical issues in pharmacokinetics, opsonization and protein-binding properties. Prog. Lipid Res., 2003, 42(6), 463-478.

[100] Moghimi, S.M.; Muir, I.S.; Illum, L.; Davis, S.S. Kolb-Bachofen, V. Coating particles with a block co-polymer (poloxamine-908) suppresses opsonization but permits the activity of dysopsonins in the serum. Biochim. Biophys. Acta., 1993, 1179(2), 157-165.

[101] Allen, T.M.; Newman, M.S.; Woodle, M.C.; Mayhew, E. Uster, P.S. Pharmacokinetics and anti-tumor activity of vincristine encapsulated in sterically stabilized liposomes. Int. J. Cancer, 1995, 62(2), 199-204.

[102] Woodle, M.C.; Matthay, K.K.; Newman, M.S.; Hidayat, J.E.; Collins, L.R. Versatility in lipid compositions showing prolonged circulation with sterically stabilized liposomes. Biochim. Biophys. Acta, 1992, 1105(2), 193-200.

[103] Gabizon, A.A. Selective tumor localization and improved therapeutic index of anthracyclines encapsulated in long-circulating liposomes. Cancer Res., 1992, 52(4), 891-896.

[104] Huang, S.K.; Lee, K.D.; Hong, K.; Friend, D.S. Papahadjopoulos, D. Microscopic localization of sterically stabilized liposomes in colon carcinoma-bearing mice. Cancer Res., 1992, 52(19), 51355143.

[105] Gabizon, A.; Chemla, M.; Tzemach, D.; Horowitz, A.T. Goren, D. Liposome longevity and stability in circulation: effects on the in vivo delivery to tumors and therapeutic efficacy of encapsulated anthracyclines. J. Drug Target., 1996, 3(5), 391-398.

[106] Webb, M.S.; Harasym, T.O.; Masin, D.; Bally, M.B. Mayer, L.D. Sphingomyelin-cholesterol liposomes significantly enhance the pharmacokinetic and therapeutic properties of vincristine in murine and human tumour models. Br. J. Cancer, 1995, 72(4), 896-904.

[107] Cabanes, A.; Tzemach, D.; Goren, D.; Horowitz, A.T. Gabizon, A. Comparative study of the antitumor activity of free doxorubicin and polyethylene glycol-coated liposomal doxorubicin in a mouse lymphoma model. Clin. Cancer Res., 1998, 4(2), 499-505.

[108] Hosoda, J.; Unezaki, S.; Maruyama, K.; Tsuchiya, S. Iwatsuru, M. Antitumor activity of doxorubicin encapsulated in poly(ethylene glycol)-coated liposomes. Biol. Pharm. Bull., 1995, 18(9), 12341237.

[109] Maillard, S.; Ameller, T.; Gauduchon, J.; Gougelet, A.; Gouilleux, F. Innovative drug delivery nanosystems improve the anti-tumor activity in vitro and in vivo of anti-estrogens in human breast cancer and multiple myeloma. J. Steroid Biochem. Mol. Biol., 2005, 94(1-3), 111-121.

[110] Maillard, S.; Gauduchon, J.; Marsaud, V.; Gouilleux, F.; Connault, E. Improved antitumoral properties of pure antiestrogen RU 58668loaded liposomes in multiple myeloma. J. Steroid Biochem. Mol. Biol., 2006, 100(1-3), 67-78.

[111] Wang, C.; Wei, Y.; Yu, L. Zhang, L. The effect of stealth liposomes on pharmacokinetics, tissue distribution and anti-tumor activity of oridonin. PDA J. Pharm. Sci. Technol., 2009, 63(5), 409-416.

[112] Zamboni, W.C.; Ramalingam, S.; Friedland, D.M.; Edwards, R.P.; Stoller, R.G. Phase I and pharmacokinetic study of pegylated liposomal CKD-602 in patients with advanced malignancies. Clin. Cancer Res., 2009, 15(4), 1466-1472.

[113] Urbinati, G.; Audisio, D.; Marsaud, V.; Plassat, V.; Arpicco, S. Therapeutic potential of new 4-hydroxy-tamoxifen-loaded $\mathrm{pH}$ gradient liposomes in a multiple myeloma experimental model. Pharm. Res., 2010, 27(2), 327-339.

[114] Drummond, D.C.; Noble, C.O.; Hayes, M.E.; Park, J.W. Kirpotin, D.B. Pharmacokinetics and in vivo drug release rates in liposomal nanocarrier development. J. Pharm. Sci., 2008, 97(11), 4696-4740.

[115] Weissmann, G.; Bloomgarden, D.; Kaplan, R.; Cohen, C.; Hoffstein, S. A general method for the introduction of enzymes, by means of immunoglobulin-coated liposomes, into lysosomes of deficient cells. Proc. Natl. Acad. Sci. U S A, 1975, 72(1), 88-92.

[116] Willis, M. Forssen, E. Ligand-targeted liposomes. Adv. Drug Deliv. Rev., 1998, 29(3), 249-271.

[117] Leserman, L.D.; Barbet, J.; Kourilsky, F. Weinstein, J.N. Targeting to cells of fluorescent liposomes covalently coupled with monoclonal antibody or protein A. Nature, 1980, 288(5791), 602-604.
[118] Martin, F.J. Papahadjopoulos, D. Irreversible coupling of immunoglobulin fragments to preformed vesicles. An improved method for liposome targeting. J. Biol. Chem., 1982, 257(1), 286-288.

[119] Martin, F.J.; Hubbell, W.L. Papahadjopoulos, D. Immunospecific targeting of liposomes to cells: a novel and efficient method for covalent attachment of Fab' fragments via disulfide bonds. Biochemistry, 1981, 20(14), 4229-4238.

[120] Maruyama, K.; Kennel, S.J. Huang, L. Lipid composition is important for highly efficient target binding and retention of immunoliposomes. Proc. Natl. Acad. Sci. US A, 1990, 87(15), 57445748 .

[121] Allen, T.M.; Brandeis, E.; Hansen, C.B.; Kao, G.Y. Zalipsky, S. A new strategy for attachment of antibodies to sterically stabilized liposomes resulting in efficient targeting to cancer cells. Biochim. Biophys. Acta, 1995, 1237(2), 99-108.

[122] Hansen, C.B.; Kao, G.Y.; Moase, E.H.; Zalipsky, S. Allen, T.M Attachment of antibodies to sterically stabilized liposomes: evaluation, comparison and optimization of coupling procedures. Biochim. Biophys. Acta, 1995, 1239(2), 133-144.

[123] Maruyama, K.; Takahashi, N.; Tagawa, T.; Nagaike, K. Iwatsuru, M. Immunoliposomes bearing polyethyleneglycol-coupled Fab' fragment show prolonged circulation time and high extravasation into targeted solid tumors in vivo. FEBS Lett., 1997, 413(1), 177180.

[124] Maruyama, K.; Ishida, O.; Takizawa, T. Moribe, K. Possibility of active targeting to tumor tissues with liposomes. Adv. Drug Deliv. Rev., 1999, 40(1-2), 89-102.

[125] Cheng, W.W. Allen, T.M. Targeted delivery of anti-CD19 liposomal doxorubicin in B-cell lymphoma: a comparison of whole monoclonal antibody, Fab' fragments and single chain Fv. J. Control Release, 2008, 126(1), 50-58.

[126] Drummond, D.C.; Hong, K.; Park, J.W.; Benz, C.C. Kirpotin, D.B. Liposome targeting to tumors using vitamin and growth factor receptors. Vitam. Horm., 2000, 60, 285-332.

[127] Gabizon, A.; Shmeeda, H.; Horowitz, A.T. Zalipsky, S. Tumor cell targeting of liposome-entrapped drugs with phospholipid-anchored folic acid-PEG conjugates. Adv. Drug Deliv. Rev., 2004, 56(8), 1177-1192.

[128] Leamon, C.P.; Low, P.S. Delivery of macromolecules into living cells: a method that exploits folate receptor endocytosis. Proc. Natl. Acad. Sci. US A, 1991, 88(13), 5572-5576.

[129] Lee, R.J. Low, P.S. Delivery of liposomes into cultured KB cells via folate receptor-mediated endocytosis. J. Biol. Chem., 1994, 269(5), 3198-3204.

[130] Pradhan, P.; Banerjee, R.; Bahadur, D.; Koch, C.; Mykhaylyk, O., et al. Targeted magnetic liposomes loaded with doxorubicin. Methods Mol. Biol., 2010, 605, 279-293.

[131] Hatakeyama, H.; Akita, H.; Maruyama, K.; Suhara, T.; Harashima, H. Factors governing the in vivo tissue uptake of transferrincoupled polyethylene glycol liposomes in vivo. Int. J. Pharm., 2004, 281(1-2), 25-33.

[132] Xu, L.; Huang, C.C.; Huang, W.; Tang, W.H.; Rait, A. Systemic tumor-targeted gene delivery by anti-transferrin receptor $\mathrm{scFv}$ immunoliposomes. Mol. Cancer Ther., 2002, 1(5), 337-346.

[133] Dagar, S.; Krishnadas, A.; Rubinstein, I.; Blend, M.J. Onyuksel, H. VIP grafted sterically stabilized liposomes for targeted imaging of breast cancer: in vivo studies. J. Control Release, 2003, 91(1-2), 123-133.

[134] Dagar, S.; Sekosan, M.; Lee, B.S.; Rubinstein, I. Onyuksel, H. VIP receptors as molecular targets of breast cancer: implications for targeted imaging and drug delivery. J. Control Release, 2001, 74(13), 129-134.

[135] Zhang, Y.F.; Wang, J.C.; Bian, D.Y.; Zhang, X. Zhang, Q. Targeted delivery of RGD-modified liposomes encapsulating both combretastatin A-4 and doxorubicin for tumor therapy: in vitro and in vivo studies. Eur. J. Pharm. Biopharm., 2010, 74(3), 467-473.

[136] Platt, V.M.; Szoka, F.C., Jr. Anticancer therapeutics: targeting macromolecules and nanocarriers to hyaluronan or CD44, a hyaluronan receptor. Mol. Pharm., 2008, 5(4), 474-486.

[137] Bourguignon, L.Y. Hyaluronan-mediated CD44 activation of RhoGTPase signaling and cytoskeleton function promotes tumor progression. Semin, Cancer Biol., 2008, 18(4), 251-259.

[138] Bourguignon, L.Y.; Spevak, C.C.; Wong, G.; Xia, W. Gilad, E. Hyaluronan-CD44 interaction with protein kinase $\mathrm{C}$ (epsilon) promotes oncogenic signaling by the stem cell marker Nanog and the Production of microRNA-21, leading to down-regulation of the 
tumor suppressor protein PDCD4, anti-apoptosis, and chemotherapy resistance in breast tumor cells. J. Biol. Chem., 2009, 284(39), 26533-26546.

[139] Lee, C.M.; Tanaka, T.; Murai, T.; Kondo, M.; Kimura, J. Novel chondroitin sulfate-binding cationic liposomes loaded with cisplatin efficiently suppress the local growth and liver metastasis of tumor cells in vivo. Cancer Res., 2002, 62(15), 4282-4288.

[140] Tseng, Y.L.; Liu, J.J. Hong, R.L. Translocation of liposomes into cancer cells by cell-penetrating peptides penetratin and tat: a kinetic and efficacy study. Mol. Pharmacol., 2002, 62(4), 864-872.

[141] Alexiou, C.; Jurgons, R.; Seliger, C. Iro, H. Medical applications of magnetic nanoparticles. J. Nanosci. Nanotechnol., 2006, 6(9-10), 2762-2768.

[142] Alexiou, C.; Schmid, R.J.; Jurgons, R.; Kremer, M.; Wanner, G., et $a l$. Targeting cancer cells: magnetic nanoparticles as drug carriers. Eur. Biophys. J., 2006, 35(5), 446-450.

[143] Martina, M.S.; Fortin, J.P.; Menager, C.; Clement, O.; Barratt, G. Generation of superparamagnetic liposomes revealed as highly efficient MRI contrast agents for in vivo imaging. J. Am. Chem. Soc., 2005, 127(30), 10676-10685.

[144] Martina, M.S.; Wilhelm, C. Lesieur, S. The effect of magnetic targeting on the uptake of magnetic-fluid-loaded liposomes by human prostatic adenocarcinoma cells. Biomaterials, 2008, 29(30), 4137-4145.

[145] Plassat, V.; Martina, M.S.; Barratt, G.; Menager, C. Lesieur, S. Sterically stabilized superparamagnetic liposomes for MR imaging and cancer therapy: pharmacokinetics and biodistribution. Int. J. Pharm., 2007, 344(1-2), 118-127.

[146] Drummond, D.C.; Marx, C.; Guo, Z.; Scott, G.; Noble, C. Enhanced pharmacodynamic and antitumor properties of a histone deacetylase inhibitor encapsulated in liposomes or ErbB2-targeted immunoliposomes. Clin. Cancer Res., 2005, 11(9), 3392-3401.

[147] Lopes de Menezes, D.E.; Pilarski, L.M.; Belch, A.R. Allen, T.M. Selective targeting of immunoliposomal doxorubicin against human multiple myeloma in vitro and ex vivo. Biochim Biophys. Acta, 2000, 1466(1-2), 205-220.

[148] Noble, C.O.; Guo, Z.; Hayes, M.E.; Marks, J.D.; Park, J.W. Characterization of highly stable liposomal and immunoliposomal formulations of vincristine and vinblastine. Cancer Chemother. Pharmacol., 2009, 64(4), 741-751.

[149] Benzinger, P.; Martiny-Baron, G.; Reusch, P.; Siemeister, G.; Kley, J.T. Targeting of endothelial KDR receptors with 3G2 immunoliposomes in vitro. Biochim. Biophys. Acta, 2000, 1466(1-2), 7178.

[150] Loomis, K.; Smith, B.; Feng, Y.; Garg, H.; Yavlovich, A. Specific targeting to B cells by lipid-based nanoparticles conjugated with a novel CD22-ScFv. Exp. Mol. Pathol., 2010, 88(2), 238-249.

[151] Kirpotin, D.B.; Drummond, D.C.; Shao, Y.; Shalaby, M.R.; Hong, $\mathrm{K}$. Antibody targeting of long-circulating lipidic nanoparticles does not increase tumor localization but does increase internalization in animal models. Cancer Res, 2006, 66(13), 6732-6740.

[152] Saad, M.; Garbuzenko, O.B.; Ber, E.; Chandna, P.; Khandare, J.J. Receptor targeted polymers, dendrimers, liposomes: which nanocarrier is the most efficient for tumor-specific treatment and imaging? J. Control Release, 2008, 130(2), 107-114.

[153] David, S.; Pitard, B.; Benoit, J.P. Passirani, C. Non-viral nanosystems for systemic siRNA delivery. Pharmacol. Res., 2009, 62(2), 100-114.

[154] Elouahabi, A. Ruysschaert, J.M. Formation and intracellular trafficking of lipoplexes and polyplexes. Mol. Ther., 2005, 11(3), 336347.

[155] Ogris, M.; Steinlein, P.; Carotta, S.; Brunner, S. Wagner, E. DNA/polyethylenimine transfection particles: influence of ligands, polymer size, and PEGylation on internalization and gene expression. AAPS PharmSci., 2001, 3(3), E21.

[156] de Lima, M.C.; Faneca, H.; Mano, M.; Penacho, N.; Duzgunes, N. Biophysical characterization of cationic liposome-DNA complexes and their interaction with cells. Methods Enzymol., 2003, 373(298312 .
[157] Simoes, S.; Pires, P.; da Cruz, M.T.; Duzgunes, N.; de Lima, M.C. Gene delivery by cationic liposome-DNA complexes containing transferrin or serum albumin. Methods Enzymol., 2003, 373, 369383.

[158] Surace, C.; Arpicco, S.; Dufay-Wojcicki, A.; Marsaud, V.; Bouclier, C. Lipoplexes targeting the CD44 hyaluronic acid receptor for efficient transfection of breast cancer cells. Mol. Pharm., 2009, 6(4), 1062-1073.

[159] Thierry, A.R.; Rahman, A. Dritschilo, A. Overcoming multidrug resistance in human tumor cells using free and liposomally encapsulated antisense oligodeoxynucleotides. Biochem. Biophys. Res. Commun., 1993, 190(3), 952-960.

[160] Brignole, C.; Pagnan, G.; Marimpietri, D.; Cosimo, E.; Allen, T.M. Targeted delivery system for antisense oligonucleotides: a novel experimental strategy for neuroblastoma treatment. Cancer Lett., 2003, 197(1-2), 231-235.

[161] Zhang, C.; Tang, N.; Liu, X.; Liang, W.; Xu, W. siRNA-containing liposomes modified with polyarginine effectively silence the targeted gene. J. Control Release, 2006, 112(2), 229-239.

[162] Taetz, S.; Bochot, A.; Surace, C.; Arpicco, S.; Renoir, J.M., et al. Hyaluronic acid-modified DOTAP/DOPE liposomes for the targeted delivery of anti-telomerase siRNA to CD44-expressing lung cancer cells. Oligonucleotides, 2009, 19(2), 103-116.

[163] Marcato, P.D. Duran, N. New aspects of nanopharmaceutical delivery systems. J. Nanosci. Nanotechnol., 2008, 8(5), 2216-2229.

[164] Lay, M.; Callejo, B.; Chang, S.; Hong, D.K.; Lewis, D.B., et al. Cationic lipid/DNA complexes (JVRS-100) combined with influenza vaccine (Fluzone) increases antibody response, cellular immunity, and antigenically drifted protection. Vaccine, 2009, 27(29), 3811-3820.

[165] Wolff, A.C.; Wang, M.; Li, H.; Pins, M.R.; Pretorius, F.J. Phase II trial of pegylated liposomal doxorubicin plus docetaxel with and without trastuzumab in metastatic breast cancer: Eastern Cooperative Oncology Group trial E3198. Breast Cancer Res. Treat., 2010, 121(1), 111-120.

[166] Sharma, A.; Mayhew, E.; Bolcsak, L.; Cavanaugh, C.; Harmon, P., et al. Activity of paclitaxel liposome formulations against human ovarian tumor xenografts. Int. J. Cancer, 1997, 71(1), 103-107.

[167] Lee, R.J. Liposomal delivery as a mechanism to enhance synergism between anticancer drugs. Mol. Cancer Ther., 2006, 5(7), 16391640 .

[168] Mayer, L.D.; Harasym, T.O.; Tardi, P.G.; Harasym, N.L.; Shew, C.R. Ratiometric dosing of anticancer drug combinations: controlling drug ratios after systemic administration regulates therapeutic activity in tumor-bearing mice. Mol. Cancer Ther., 2006, 5(7), 1854-1863.

[169] Wang, J.; Goh, B.; Lu, W.; Zhang, Q.; Chang, A. In vitro cytotoxicity of Stealth liposomes co-encapsulating doxorubicin and verapamil on doxorubicin-resistant tumor cells. Biol. Pharm. Bull., 2005, 28(5), 822-828.

[170] Matsumura, Y. Maeda, H. A new concept for macromolecular therapeutics in cancer chemotherapy: mechanism of tumoritropic accumulation of proteins and the antitumor agent smancs. Cancer Res., 1986, 46(12 Pt 1), 6387-6392.

[171] Fanciullino, R. Ciccolini, J. Liposome-encapsulated anticancer drugs: still waiting for the magic bullet? Curr. Med. Chem., 2009 16(33), 4361-4371.

[172] Muggia, F.M.; Hainsworth, J.D.; Jeffers, S.; Miller, P.; Groshen, S. Phase II study of liposomal doxorubicin in refractory ovarian cancer: antitumor activity and toxicity modification by liposomal encapsulation. J. Clin. Oncol., 1997, 15(3), 987-993.

[173] Jiskoot, W.; van Schie, R.M.; Carstens, M.G. Schellekens, H. Immunological risk of injectable drug delivery systems. Pharm. Res., 2009, 26(6), 1303-1314.

[174] Semple, S.C.; Harasym, T.O.; Clow, K.A.; Ansell, S.M.; Klimuk, S.K. Immunogenicity and rapid blood clearance of liposomes containing polyethylene glycol-lipid conjugates and nucleic Acid. $J$. Pharmacol. Exp. Ther., 2005, 312(3), 1020-1026. 